\title{
THRESHOLD APPROXIMATIONS FOR A FACTORIZED SELFADJOINT OPERATOR FAMILY WITH THE FIRST AND SECOND CORRECTORS TAKEN INTO ACCOUNT
}

\author{
E. S. VASILEVSKAYA AND T. A. SUSLINA \\ Dedicated to Vasiliı Mikhaìlovich Babich \\ on the occasion of his birthday
}

\begin{abstract}
In a Hilbert space $\mathfrak{H}$, a family of operators $A(t)$ admitting a factorization of the form $A(t)=X(t)^{*} X(t)$, where $X(t)=X_{0}+t X_{1}, t \in \mathbb{R}$, is considered. It is assumed that the point $\lambda_{0}=0$ is an isolated eigenvalue of finite multiplicity for $A(0)$. Let $F(t)$ be the spectral projection of $A(t)$ for the interval $[0, \delta]$ (where $\delta$ is sufficiently small). For small $|t|$, approximations in the operator norm in $\mathfrak{H}$ are obtained for the projection $F(t)$ with an error of $O\left(|t|^{3}\right)$ and for the operator $A(t) F(t)$ with an error of $O\left(|t|^{5}\right)$ (the threshold approximations). By using these results, approximation in the operator norm in $\mathfrak{H}$ are constructed for the operator exponential $\exp (-A(t) \tau)$ for large $\tau>0$ with an error of $O\left(\tau^{-3 / 2}\right)$. For the resolvent $\left(A(t)+\varepsilon^{2} I\right)^{-1}$ multiplied by a suitable "smoothing" factor, approximation in the operator norm in $\mathfrak{H}$ for small $\varepsilon>0$ with an error of $O(\varepsilon)$ is obtained. All approximations are given in terms of the spectral characteristics of $A(t)$ near the bottom of the spectrum. In these approximations, the first and the second correctors are taken into account. The results are aimed at applications to homogenization problems for periodic differential operators in the small period limit.
\end{abstract}

\section{INTRODUCTION}

0.1. In a series of papers [BSu1, BSu2, BSu3, BSu4] by M. Sh. Birman and T. A. Suslina, a new operator-theoretic approach to the problems of homogenization in the small period limit was suggested. By this approach, the so-called operator error estimates in homogenization theory were obtained. In [BSu1, BSu2, BSu3, BSu4, the new approach was applied to elliptic homogenization problems, and in [Su1, $\mathrm{Su2}$, VV, Su3, Su4] it was applied to parabolic problems. Under this method, after using a scaling transformation and the Floquet-Bloch decomposition, a family of differential operators depending on the parameter (the quasimomentum) arises. It is convenient to study this operator family in the framework of an abstract operator-theoretic pattern.

The present paper is a further development of the corresponding operator-theoretic material. At the same time, this material is of interest by its own. The authors are going to devote a separate paper to application of the results obtained here to homogenization problems.

In the abstract framework, a family of selfadjoint operators $A(t)$ acting in a separable Hilbert space $\mathfrak{H}$ is considered. This family admits a factorization of the form $A(t)=$ $X(t)^{*} X(t)$, where $X(t)=X_{0}+t X_{1}, t \in \mathbb{R}$. It is assumed that the point $\lambda_{0}=0$ is an

2010 Mathematics Subject Classification. Primary 47A55.

Key words and phrases. Analytic perturbation theory, threshold approximations, corrector.

Supported by RFBR (grant no. 08-01-00209-a) and the Program of support of the leading scientific schools (grant NSh-5931.2010.1). 
isolated eigenvalue of finite multiplicity $n$ for the operator $A(0)$. Let $\mathfrak{N}=\operatorname{Ker} A(0)$, and let $P$ be the orthogonal projection of $\mathfrak{H}$ onto the subspace $\mathfrak{N}$.

The family $A(t)$ is an analytic operator-valued function of $t$. We study the spectral characteristics of $A(t)$ near the bottom of its spectrum by methods of analytic perturbation theory. The above factorization of the operator $A(t)$ allows us to advance far.

Fix a sufficiently small number $\delta>0$. If $t^{0}$ is sufficiently small and $|t| \leq t^{0}$, then the operator $A(t)$ has exactly $n$ eigenvalues in the interval $[0, \delta]$. (The values of $\delta$ and $t^{0}$ are controlled; see $\S 1$.) By the analytic perturbation theory, there exist real-analytic branches of the eigenvalues $\lambda_{l}(t)$ and real-analytic branches of orthonormal eigenvectors $\varphi_{l}(t), l=1, \ldots, n$, of the operator $A(t)$. Herewith, $\lambda_{l}(t)=\gamma_{l} t^{2}+O\left(|t|^{3}\right)$, where $\gamma_{l} \geq 0$, and $\varphi_{l}(t)=\omega_{l}+O(|t|), l=1, \ldots, n$. The vectors $\omega_{1}, \ldots, \omega_{n}$ form an orthonormal basis in $\mathfrak{N}$. The crucial role is played by the so-called spectral germ of the operator family $A(t)$ at $t=0$ : this is a selfadjoint operator $S: \mathfrak{N} \rightarrow \mathfrak{N}$ such that $S \omega_{l}=\gamma_{l} \omega_{l}, l=1, \ldots, n$. By using a factorization for the operator $A(t)$, an invariant definition of the spectral germ can be given (see $\S 1$ ).

Let $F(t)$ be the spectral projection of $A(t)$ for the interval $[0, \delta]$. Our first goal is to find approximations in the operator norm in $\mathfrak{H}$ for the operators $F(t)$ and $A(t) F(t)$ for $|t| \leq t^{0}$ (the so-called threshold approximations). In [BSu1, Chapter 1], the principal terms of such approximations with the following error estimates were found:

$$
\|F(t)-P\|_{\mathfrak{H} \rightarrow \mathfrak{H}} \leq C_{1}|t|, \quad\left\|A(t) F(t)-t^{2} S P\right\|_{\mathfrak{H} \rightarrow \mathfrak{H}} \leq C_{2}|t|^{3}, \quad|t| \leq t^{0} .
$$

In [BSu2], sharper approximations for $F(t)$ with an error of $O\left(t^{2}\right)$ and for $A(t) F(t)$ with an error of $O\left(t^{4}\right)$ were obtained. In the present paper, we establish still sharper approximations for $F(t)$ with an error of $O\left(|t|^{3}\right)$ and for $A(t) F(t)$ with an error of $O\left(|t|^{5}\right)$. These approximations are given in terms of finite rank operators arising in the analytic perturbation theory.

As in the previous publications, in order to calculate the approximate operators we use the power series expansions for analytic branches of eigenvalues and eigenvectors of $A(t)$, while in order to prove error estimates we integrate the difference of the resolvents of $A(t)$ and $A(0)$ over a suitable contour in the complex plane.

0.2. The resulting approximations for the operators $F(t)$ and $A(t) F(t)$ are applied to the problems of approximation in the operator norm in $\mathfrak{H}$ for operator-valued functions. Namely, we approximate the operator exponential $\exp (-A(t) \tau)$ for large $\tau>0$ and the resolvent $\left(A(t)+\varepsilon^{2} I\right)^{-1}$ for small $\varepsilon>0$. It is assumed that for some $c_{*}>0$ we have $A(t) \geq c_{*} t^{2} I$ for $|t| \leq t^{0}$; this ensures that the spectral germ $S$ is nondegenerate.

Let us discuss the results for the operator exponential. In [Su1, Su2], the principal term of approximation for the operator exponential $\exp (-A(t) \tau)$ was obtained by using estimates (0.1). This approximation is given in terms of the (finite rank) operator $\exp \left(-t^{2} S \tau\right) P$; the error estimate is of order $|\tau|^{-1 / 2}$. Next, in [V], on the basis of the results of $\mathrm{BSu} 2$, a more accurate approximation for the exponential $\exp (-A(t) \tau)$ with an error of order $|\tau|^{-1}$ was obtained. In this approximation, the first corrector (of order $\left.O\left(|\tau|^{-1 / 2}\right)\right)$ was taken into account.

In the present paper, we obtain even more accurate approximation of the exponential $\exp (-A(t) \tau)$ with an error of order $|\tau|^{-3 / 2}$. For this, we take the sum of the first and second correctors into account (the second corrector is of order $O\left(|\tau|^{-1}\right)$ ).

Note that the norm of the operator $\exp (-A(t) \tau) F(t)^{\perp}$ is estimated by $e^{-\delta \tau}$ and "moves to the error" with reserve. Therefore, the problem reduces to approximation of the operator $\exp (-A(t) \tau) F(t)$. This makes if possible to apply the threshold approximations for $F(t)$ and $A(t) F(t)$. This argument shows that the problem under investigation is of "threshold nature". 
0.3. Now we discuss the results for the resolvent $\left(A(t)+\varepsilon^{2} I\right)^{-1}$. In BSu1, Chapter 1], estimates (0.1) were employed to find the principal term of approximation for this resolvent in terms of the operator $\left(t^{2} S+\varepsilon^{2} I\right)^{-1} P$ with an error of order $O\left(\varepsilon^{-1}\right)$. (Note that the resolvent $\left(A(t)+\varepsilon^{2} I\right)^{-1}$ itself is of order $O\left(\varepsilon^{-2}\right)$.) In BSu2, a more accurate approximation for the resolvent $\left(A(t)+\varepsilon^{2} I\right)^{-1}$ with an error of order $O(1)$ was obtained. Herewith, the first corrector (of order $\varepsilon^{-1}$ ) was taken into account.

Note that the norm of the operator $\left(A(t)+\varepsilon^{2} I\right)^{-1} F(t)^{\perp}$ is estimated by the constant $\delta^{-1}$ and "moves to the error" if the resolvent is approximated with an error of $O(1)$. This shows the "threshold nature" of the problem.

However, if one looks for even more accurate approximation of the resolvent with an error of $O(\varepsilon)$, then the term $\left(A(t)+\varepsilon^{2} I\right)^{-1} F(t)^{\perp}$ does not move to the error any longer. This is an essential difference between the elliptic and the parabolic problems. In order to obtain approximation of the resolvent with an error of $O(\varepsilon)$, we multiply the resolvent $\left(A(t)+\varepsilon^{2} I\right)^{-1}$ by a suitable "smoothing factor" from the right. (In applications to differential operators, the corresponding operator is smoothing indeed, allowing us to transfer this term to the abstract context.) We obtain approximation of the "smoothed" resolvent $\left(A(t)+\varepsilon^{2} I\right)^{-1}$ with an error of $O(\varepsilon)$. Now, the sum of the first and the second correctors is taken into account (the second corrector is of order $O(1)$ ).

0.4. The structure of the paper. In $\S 1$, we introduce the operator family $A(t)$ and some auxiliary operators (including the spectral germ) arising in considerations in the spirit of perturbation theory. Also, we consider power series expansions for analytic branches of eigenvalues and eigenvectors of the operator $A(t)$. In $\S 2$, the power series expansions for the operators $F(t)$ and $A(t) F(t)$ are treated and several terms of these expansions are calculated. In $\S 3$, we find a fairly sharp approximation for the difference of the resolvents of the operators $A(t)$ and $A(0)$ on a suitable contour in the complex plane. Applying the results of $\S 3$, in $\S 4$ we prove error estimates in approximations for $F(t)$ and $A(t) F(t) . \quad \S 5$ is devoted to approximation of the operator exponential $\exp (-A(t) \tau)$ for large values of $\tau$. In $\S 6$, the case of an operator family of a special form, namely, $A(t)=M^{*} \widehat{A}(t) M$, is considered; approximation for the operator $M e^{-A(t) \tau} M^{*}$ (a sandwiched operator exponential) is obtained. $\S 7$ is devoted to approximation of the resolvent $\left(A(t)+\varepsilon^{2} I\right)^{-1}$ multiplied by a suitable "smoothing" factor.

Formally, the paper can be read without consulting the previous papers. All necessary facts are duplicated (some of them with proofs). At the same time, the acquaintance with [BSu1, Chapter 1], [BSu2, [Su2, §2], [V] §2, 3] may facilitate reading.

0.5. Notation. Let $\mathcal{G}$ and $\mathcal{G}_{*}$ be separable Hilbert spaces. The symbols $(\cdot, \cdot)_{\mathcal{G}}$ and $\|\cdot\|_{\mathcal{G}}$ stand for the inner product and the norm in $\mathcal{G}$, respectively; the symbol $\|\cdot\|_{\mathcal{G} \rightarrow \mathcal{G}_{*}}$ stands for the norm of a continuous linear operator from $\mathcal{G}$ to $\mathcal{G}_{*}$. Sometimes we omit indices if this does not lead to confusion. By $I=I_{\mathcal{G}}$ we denote the identity operator in $\mathcal{G}$. If $A: \mathcal{G} \rightarrow \mathcal{G}_{*}$ is a linear operator, then $\operatorname{Dom} A$ denotes the domain of $A$ and Ker $A$ denotes the kernel of $A$. If $\mathfrak{N}$ is a subspace in $\mathcal{G}$, then $\mathfrak{N}^{\perp}:=\mathcal{G} \ominus \mathfrak{N}$. If $P$ is the orthogonal projection of $\mathcal{G}$ onto $\mathfrak{N}$, then $P^{\perp}$ is the orthogonal projection onto $\mathfrak{N}^{\perp}$.

By $\beta$ with various indices and marks we denote absolute constants. Other (specific) constants in estimates are denoted by $c, C, \mathcal{C}, \mathfrak{C}$ with various indices and marks.

\section{§1. A FACTORIZED FAMILY OF SELFADJOINT OPERATORS}

The content of Subsections 1.1, 1.2, 1.3, and 1.5 is borrowed from [BSu1, Chapter 1], BSu2]. 
1.1. The family of operators $A(t)$. Let $\mathfrak{H}$ and $\mathfrak{H}_{*}$ be separable complex Hilbert spaces, let $X_{0}: \mathfrak{H} \rightarrow \mathfrak{H}_{*}$ be a densely defined and closed operator, and let $X_{1}: \mathfrak{H} \rightarrow \mathfrak{H}_{*}$ be a bounded operator. On the domain $\operatorname{Dom} X(t)=\operatorname{Dom} X_{0}$, we consider the operator $X(t):=X_{0}+t X_{1}, t \in \mathbb{R}$. Our main object is a family of selfadjoint (and nonnegative) operators

$$
A(t):=X(t)^{*} X(t), \quad t \in \mathbb{R}
$$

in the space $\mathfrak{H}$. We denote $A(0)=X_{0}^{*} X_{0}=: A_{0}$. The operator (1.1) corresponds to the quadratic form $a(t)[u, u]=\|X(t) u\|_{\mathfrak{H}_{*}}^{2}, u \in \operatorname{Dom} X_{0}$. We denote

$$
\mathfrak{N}:=\operatorname{Ker} A_{0}=\operatorname{Ker} X_{0}, \quad \mathfrak{N}_{*}:=\operatorname{Ker} X_{0}^{*} .
$$

It is assumed that the number $\lambda_{0}=0$ is an isolated point of the spectrum of $A_{0}$, and that

$$
0<n:=\operatorname{dim} \mathfrak{N}<\infty, \quad n \leq n_{*}:=\operatorname{dim} \mathfrak{N}_{*} \leq \infty .
$$

The distance from the point $\lambda_{0}=0$ to the rest of the spectrum of $A_{0}$ is denoted by $d^{0}$.

Let $F(t, s)$ be the spectral projection of the operator $A(t)$ for an interval $[0, s]$. In what follows, we fix a number $\delta>0$ such that $8 \delta<d^{0}$. It turns out (see BSu1, Chapter 1 , (1.3)]) that

$$
F(t, \delta)=F(t, 3 \delta), \quad \operatorname{rank} F(t, \delta)=n, \quad|t| \leq t^{0}=\delta^{1 / 2}\left\|X_{1}\right\|^{-1} .
$$

Below we write $F(t)=F(t, \delta)$. Let $P$ be the orthogonal projection of the space $\mathfrak{H}$ onto the subspace $\mathfrak{N}$, and let $P_{*}$ be the orthogonal projection of $\mathfrak{H}_{*}$ onto $\mathfrak{N}_{*}$. We use the notation $P^{\perp}=I-P, \mathfrak{N}^{\perp}=\mathfrak{H} \ominus \mathfrak{N}$, etc.

The operator family $A(t)$ depends on the parameter $t$ analytically; the point $\lambda_{0}=0$ is an isolated eigenvalue of finite multiplicity $n$ for the unperturbed operator $A_{0}$. Therefore, for small $|t|$ the eigenvalues of the operator $A(t)$ near zero and the corresponding eigenvectors can be studied by methods of the analytic perturbation theory.

1.2. The operators $Z$ and $R$. We introduce some auxiliary operators that arise in the study of $A(t)$ in the framework of the analytic perturbation theory.

Let $\mathcal{D}:=\operatorname{Dom} X_{0} \cap \mathfrak{N}^{\perp}$. The form $\left(X_{0} \eta, X_{0} \zeta\right)_{\mathfrak{H}_{*}}, \eta, \zeta \in \mathcal{D}$, determines an inner product in $\mathcal{D}$ converting $\mathcal{D}$ into a Hilbert space. We have

$$
\left\|X_{0} \zeta\right\|_{\mathfrak{H}_{*}}^{2} \geq d^{0}\|\zeta\|_{\mathfrak{H}}^{2} \geq 8 \delta\|\zeta\|_{\mathfrak{H}}^{2}, \quad \zeta \in \mathcal{D} .
$$

Let $u \in \mathfrak{H}_{*}$. The equation $X_{0}^{*}\left(X_{0} \psi-u\right)=0$ for an element $\psi \in \mathcal{D}$ will be understood in the weak sense:

$$
\left(X_{0} \psi, X_{0} \zeta\right)_{\mathfrak{H}_{*}}=\left(u, X_{0} \zeta\right)_{\mathfrak{H}_{*}}, \quad \zeta \in \mathcal{D}
$$

(cf. [BSu1, Chapter 1, (1.7)]). The right-hand side of (1.4) is an antilinear continuous functional of $\zeta \in \mathcal{D}$. By the Riesz theorem, there exists a unique solution $\psi$, and $\left\|X_{0} \psi\right\|_{\mathfrak{H}_{*}} \leq\|u\|_{\mathfrak{H}_{*}}$. Now, let $\omega \in \mathfrak{N}$ and $u=-X_{1} \omega$, i.e.,

$$
X_{0}^{*}\left(X_{0} \psi+X_{1} \omega\right)=0 .
$$

The solution of (1.5) is denoted by $\psi(\omega)$. We define a bounded operator $Z: \mathfrak{H} \rightarrow \mathfrak{H}$ by the relations

$$
Z \omega=\psi(\omega), \quad \omega \in \mathfrak{N} ; \quad Z x=0, \quad x \in \mathfrak{N}^{\perp}
$$

(cf. [BSu2, Subsection 1.2]). Clearly, $\operatorname{rank} Z \leq n, Z P=Z$, and $P Z=0$. By (1.3),

$$
8 \delta\|\psi(\omega)\|_{\mathfrak{H}}^{2} \leq\left\|X_{0} \psi(\omega)\right\|_{\mathfrak{H}_{*}}^{2} \leq\left\|X_{1} \omega\right\|_{\mathfrak{H}_{*}}^{2} \leq\left\|X_{1}\right\|^{2}\|\omega\|_{\mathfrak{H}}^{2}
$$

whence

$$
\|Z\| \leq(8 \delta)^{-1 / 2}\left\|X_{1}\right\|
$$


Now, we introduce a linear operator $R$ (see [BSu1, Chapter 1, Subsection 1.2]) as follows:

$$
R: \mathfrak{N} \rightarrow \mathfrak{N}_{*}, \quad R \omega=X_{0} Z \omega+X_{1} \omega .
$$

Another description of $R$ is given by the formula $R=\left.P_{*} X_{1}\right|_{\mathfrak{N}}$, which implies the estimate

$$
\|R\| \leq\left\|X_{1}\right\| \text {. }
$$

1.3. The spectral germ. In accordance with [BSu1, Subsection 1.3], the operator

$$
S=R^{*} R: \mathfrak{N} \rightarrow \mathfrak{N}
$$

is called the spectral germ of the operator family (1.1) at $t=0$. The germ can be written as $S=\left.P X_{1}^{*} P_{*} X_{1}\right|_{\mathfrak{N}}$ and admits the estimate

$$
\|S\| \leq\left\|X_{1}\right\|^{2} .
$$

The germ $S$ is said to be nondegenerate if $\operatorname{Ker} S=\{0\}$, or equivalently, rank $R=n$. The spectral meaning of the operator $S$ is discussed below in Subsection 1.5.

1.4. The operators $Z_{2}$ and $R_{2}$. Let $\omega \in \mathfrak{N}$. Consider the following equation for $\phi \in \mathcal{D}:$

$$
X_{0}^{*}\left(X_{0} \phi+X_{1} Z \omega\right)=-P^{\perp} X_{1}^{*} R \omega .
$$

The right-hand side of (1.12) belongs to $\mathfrak{N}^{\perp}=\operatorname{Ran} X_{0}^{*}$, i.e., the solvability condition is fulfilled. Equation (1.12) is understood in the weak sense:

$$
\left(X_{0} \phi, X_{0} \zeta\right)_{\mathfrak{H}_{*}}=-\left(X_{1} Z \omega, X_{0} \zeta\right)_{\mathfrak{H}_{*}}-\left(X_{1}^{*} R \omega, \zeta\right)_{\mathfrak{H}}, \quad \zeta \in \mathcal{D}
$$

The left-hand side is the inner product of the elements $\phi$ and $\zeta$ in $\mathcal{D}$, while the righthand side is an antilinear continuous functional of $\zeta \in \mathcal{D}$. By (1.3), (1.7), and (1.9), the right-hand side is estimated as follows:

$$
\begin{aligned}
\left|\left(X_{1} Z \omega, X_{0} \zeta\right)_{\mathfrak{H}_{*}}+\left(X_{1}^{*} R \omega, \zeta\right)_{\mathfrak{H}}\right| & \leq\left\|X_{1} Z \omega\right\|_{\mathfrak{H}_{*}}\left\|X_{0} \zeta\right\|_{\mathfrak{H}_{*}}+\left\|X_{1}^{*} R \omega\right\|_{\mathfrak{H}}\|\zeta\|_{\mathfrak{H}} \\
& \leq\left(\left\|X_{1}\right\|\|Z\|+\left\|X_{1}\right\|\|R\|(8 \delta)^{-1 / 2}\right)\|\omega\|_{\mathfrak{H}}\left\|X_{0} \zeta\right\|_{\mathfrak{H}_{*}} \\
& \leq(2 \delta)^{-1 / 2}\left\|X_{1}\right\|^{2}\|\omega\|_{\mathfrak{H}}\left\|X_{0} \zeta\right\|_{\mathfrak{H}_{*}} .
\end{aligned}
$$

By the Riesz theorem, there exists a unique solution $\phi=\phi(\omega)$ of equation (1.12), and

$$
\left\|X_{0} \phi(\omega)\right\|_{\mathfrak{H}_{*}} \leq(2 \delta)^{-1 / 2}\left\|X_{1}\right\|^{2}\|\omega\|_{\mathfrak{H}} .
$$

We define an operator $Z_{2}: \mathfrak{H} \rightarrow \mathfrak{H}$ by the relations

$$
Z_{2} \omega=\phi(\omega), \quad \omega \in \mathfrak{N} ; \quad Z_{2} x=0, \quad x \in \mathfrak{N}^{\perp} .
$$

Clearly, $Z_{2} P=Z_{2}$ and $P Z_{2}=0$. From (1.14) and (1.3) it follows that

$$
\left\|Z_{2}\right\| \leq(4 \delta)^{-1}\left\|X_{1}\right\|^{2} \text {. }
$$

Now, we introduce an operator $R_{2}$ as follows:

$$
R_{2}: \mathfrak{N} \rightarrow \mathfrak{H}_{*}, \quad R_{2} \omega=X_{0} Z_{2} \omega+X_{1} Z \omega .
$$

To estimate the norm of $R_{2}$, we observe that (1.13) with $\zeta=\phi(\omega)$ implies

$$
\begin{aligned}
\left\|R_{2} \omega\right\|_{\mathfrak{H}_{*}}^{2} & =\left\|X_{0} \phi(\omega)+X_{1} Z \omega\right\|_{\mathfrak{H}_{*}}^{2}=\left(R_{2} \omega, X_{1} Z \omega\right)_{\mathfrak{H}_{*}}-\left(X_{1}^{*} R \omega, \phi(\omega)\right)_{\mathfrak{H}} \\
& \leq \frac{1}{2}\left\|R_{2} \omega\right\|_{\mathfrak{H}_{*}}^{2}+\frac{1}{2}\left\|X_{1} Z \omega\right\|_{\mathfrak{H}_{*}}^{2}+\left\|X_{1}^{*} R \omega\right\|_{\mathfrak{H}}\left\|Z_{2} \omega\right\|_{\mathfrak{H}} .
\end{aligned}
$$

By (1.7), (1.9), and (1.16), we have

$$
\left\|R_{2}\right\| \leq \sqrt{5}(8 \delta)^{-1 / 2}\left\|X_{1}\right\|^{2} .
$$


1.5. Power series expansions for eigenvalues and eigenvectors. The general analytic perturbation theory (see $[\mathrm{K}]$ ) shows that for $|t| \leq t^{0}$ there exist real-analytic functions $\lambda_{l}(t)$ (the branches of eigenvalues) and real-analytic $\mathfrak{H}$-valued functions $\varphi_{l}(t)$ (the branches of eigenvectors) such that

$$
A(t) \varphi_{l}(t)=\lambda_{l}(t) \varphi_{l}(t), \quad l=1, \ldots, n, \quad|t| \leq t^{0},
$$

and the $\varphi_{l}(t), l=1, \ldots, n$, form an orthonormal basis in the space $F(t) \mathfrak{H}$. Then

$$
\left(\varphi_{l}(t), \varphi_{k}(t)\right)_{\mathfrak{H}}=\delta_{l k}, \quad l, k=1, \ldots, n, \quad|t| \leq t^{0} .
$$

For sufficiently small $t_{*} \leq t^{0}$ and $|t| \leq t_{*}$, we have the following convergent power series expansions:

$$
\begin{aligned}
& \lambda_{l}(t)=\gamma_{l} t^{2}+\mu_{l} t^{3}+\nu_{l} t^{4}+\cdots, \quad \gamma_{l} \geq 0, \quad \mu_{l}, \nu_{l} \in \mathbb{R}, \quad l=1, \ldots, n, \\
& \varphi_{l}(t)=\omega_{l}+t \varphi_{l}^{(1)}+t^{2} \varphi_{l}^{(2)}+t^{3} \varphi_{l}^{(3)}+\cdots, \quad l=1, \ldots, n .
\end{aligned}
$$

The elements $\omega_{l}=\varphi_{l}(0), l=1, \ldots, n$, form an orthonormal basis in $\mathfrak{N}$. Hence,

$$
P=\sum_{l=1}^{n}\left(\cdot, \omega_{l}\right)_{\mathfrak{H}} \omega_{l} .
$$

Relations (1.19) mean that

$$
\left(X(t) \varphi_{l}(t), X(t) \zeta\right)_{\mathfrak{H}_{*}}=\lambda_{l}(t)\left(\varphi_{l}(t), \zeta\right)_{\mathfrak{H}}, \quad \zeta \in \operatorname{Dom} X_{0}, \quad l=1, \ldots, n
$$

We substitute expansions (1.21) and (1.22) in (1.24) and compare the coefficients of the same powers of $t$. The coefficients of $t^{0}$ are equal because $\omega_{l} \in \mathfrak{N}$. Comparing the coefficients of $t^{1}$, we arrive at the equation $X_{0}^{*}\left(X_{0} \varphi_{l}^{(1)}+X_{1} \omega_{l}\right)=0$ (understood in the weak sense). By the definition of the operator $Z$ (see (1.5), (1.6)), this means that $\widetilde{\omega}_{l}:=\varphi_{l}^{(1)}-Z \omega_{l} \in \mathfrak{N}$, i.e.,

$$
\varphi_{l}^{(1)}=Z \omega_{l}+\widetilde{\omega}_{l}, \quad \widetilde{\omega}_{l} \in \mathfrak{N}, \quad l=1, \ldots, n .
$$

By the definition of the operator $R$ (see (1.8)), we have

$$
X_{0} \varphi_{l}^{(1)}+X_{1} \omega_{l}=R \omega_{l}, \quad l=1, \ldots, n .
$$

Comparing the terms with $t^{2}$, we arrive at the relation

$$
\left(X_{0} \varphi_{l}^{(2)}+X_{1} \varphi_{l}^{(1)}, X_{0} \zeta\right)_{\mathfrak{H}_{*}}+\left(X_{0} \varphi_{l}^{(1)}+X_{1} \omega_{l}, X_{1} \zeta\right)_{\mathfrak{H}_{*}}=\gamma_{l}\left(\omega_{l}, \zeta\right)_{\mathfrak{H}}, \quad \zeta \in \operatorname{Dom} X_{0}
$$

If $\zeta \in \mathfrak{N}$, then (1.27) shows that

$$
\left(R \omega_{l}, X_{1} \zeta\right)_{\mathfrak{H}_{*}}=\gamma_{l}\left(\omega_{l}, \zeta\right)_{\mathfrak{H}}, \quad \zeta \in \mathfrak{N}, \quad l=1, \ldots, n .
$$

Since $X_{0}^{*} R=0$, the left-hand side can be rewritten as

$$
\left(R \omega_{l}, X_{1} \zeta\right)_{\mathfrak{H}_{*}}=\left(R \omega_{l}, X_{0} Z \zeta+X_{1} \zeta\right)_{H \mathfrak{H}_{*}}=\left(R \omega_{l}, R \zeta\right)_{\mathfrak{H}_{*}}=\left(S \omega_{l}, \zeta\right)_{\mathfrak{H}} .
$$

Thus,

$$
\left(S \omega_{l}, \zeta\right)_{\mathfrak{H}}=\gamma_{l}\left(\omega_{l}, \zeta\right)_{\mathfrak{H}}, \quad \zeta \in \mathfrak{N}, \quad l=1, \ldots, n
$$

So, the coefficients $\gamma_{l}$ and the elements $\omega_{l}$ are eigenvalues and eigenvectors of the spectral germ $S$ :

$$
S \omega_{l}=\gamma_{l} \omega_{l}, \quad l=1, \ldots, n
$$

(cf. BSu1, Chapter 1, Proposition 1.6]). Relations (1.29) clarify the spectral meaning of the operator $S$. By (1.23) and (1.29), we have

$$
S P=\sum_{l=1}^{n} \gamma_{l}\left(\cdot, \omega_{l}\right)_{\mathfrak{H}} \omega_{l} .
$$


1.6. Calculation of the elements $\varphi_{l}^{(2)}$. Now we return to identity (1.27). Taking (1.26) into account, we rewrite (1.27) as

$$
\left(X_{0} \varphi_{l}^{(2)}+X_{1} \varphi_{l}^{(1)}, X_{0} \zeta\right)_{\mathfrak{H}_{*}}=-\left(R \omega_{l}, X_{1} \zeta\right)_{\mathfrak{H}_{*}}+\gamma_{l}\left(\omega_{l}, \zeta\right)_{\mathfrak{H}}, \quad \zeta \in \operatorname{Dom} X_{0}
$$

By (1.28),

$$
\left(R \omega_{l}, X_{1} \zeta\right)_{\mathfrak{H}_{*}}=\left(R \omega_{l}, X_{1} P \zeta\right)_{\mathfrak{H}_{*}}+\left(R \omega_{l}, X_{1} P^{\perp} \zeta\right)_{\mathfrak{H}_{*}}=\gamma_{l}\left(\omega_{l}, P \zeta\right)_{\mathfrak{H}}+\left(R \omega_{l}, X_{1} P^{\perp} \zeta\right)_{\mathfrak{H}_{*}}
$$

Since $\omega_{l} \in \mathfrak{N}$, we have $\left(\omega_{l}, P \zeta\right)_{\mathfrak{H}}=\left(\omega_{l}, \zeta\right)_{\mathfrak{H}}$, and (1.31) takes the form

$$
\left(X_{0} \varphi_{l}^{(2)}+X_{1} \varphi_{l}^{(1)}, X_{0} \zeta\right)_{\mathfrak{H}_{*}}=-\left(P^{\perp} X_{1}^{*} R \omega_{l}, \zeta\right)_{\mathfrak{H}_{*}}, \quad \zeta \in \operatorname{Dom} X_{0}
$$

For $\zeta \in \mathfrak{N}$ relation (1.32) is valid automatically (the two sides are equal to zero). Therefore, it suffices to consider $\zeta \in \mathcal{D}$. By (1.25) and (1.8),

$$
X_{1} \varphi_{l}^{(1)}=X_{1} Z \omega_{l}+X_{1} \widetilde{\omega}_{l}=R \widetilde{\omega}_{l}-X_{0} Z \widetilde{\omega}_{l}+X_{1} Z \omega_{l} .
$$

Since $\left(R \widetilde{\omega}_{l}, X_{0} \zeta\right)_{\mathfrak{H}_{*}}=0,(1.32)$ takes the form

$$
\left(X_{0}\left(\varphi_{l}^{(2)}-Z \widetilde{\omega}_{l}\right)+X_{1} Z \omega_{l}, X_{0} \zeta\right)_{\mathfrak{H}_{*}}=-\left(X_{1}^{*} R \omega_{l}, \zeta\right)_{\mathfrak{H}_{*}}, \quad \zeta \in \mathcal{D} .
$$

Combining (1.33) and the definition of $Z_{2}$ (see (1.13), (1.15)), we conclude that $\varphi_{l}^{(2)}-$ $Z \widetilde{\omega}_{l}-Z_{2} \omega_{l}=: \widetilde{\omega}_{l}^{(2)} \in \mathfrak{N}$, i.e.,

$$
\varphi_{l}^{(2)}=Z_{2} \omega_{l}+Z \widetilde{\omega}_{l}+\widetilde{\omega}_{l}^{(2)}, \quad \widetilde{\omega}_{l}^{(2)} \in \mathfrak{N}, \quad l=1, \ldots, n .
$$

By the definitions of the operators $R$ and $R_{2}$ (see (1.8) and (1.17)), relations (1.25) and (1.34) imply the identities

$$
X_{0} \varphi_{l}^{(2)}+X_{1} \varphi_{l}^{(1)}=R_{2} \omega_{l}+R \widetilde{\omega}_{l}, \quad l=1, \ldots, n .
$$

Now we substitute the expansions (1.22) in (1.20) and compare the coefficients of the same powers of $t$. The coefficients of $t^{0}$ are equal because the elements $\omega_{1}, \ldots, \omega_{n}$ are orthogonal and normalized. Comparing the coefficients of $t^{1}$, we see that

$$
\left(\varphi_{l}^{(1)}, \omega_{k}\right)_{\mathfrak{H}}+\left(\omega_{l}, \varphi_{k}^{(1)}\right)_{\mathfrak{H}}=0, \quad l, k=1, \ldots, n .
$$

Taking (1.25) and the relation $Z \omega_{l} \in \mathfrak{N}^{\perp}$ into account, we obtain

$$
\left(\widetilde{\omega}_{l}, \omega_{k}\right)_{\mathfrak{H}}+\left(\omega_{l}, \widetilde{\omega}_{k}\right)_{\mathfrak{H}}=0, \quad l, k=1, \ldots, n
$$

(cf. [BSu2, (1.18)]). Comparing the coefficients of $t^{2}$, we arrive at

$$
\left(\varphi_{l}^{(2)}, \omega_{k}\right)_{\mathfrak{H}}+\left(\varphi_{l}^{(1)}, \varphi_{k}^{(1)}\right)_{\mathfrak{H}}+\left(\omega_{l}, \varphi_{k}^{(2)}\right)_{\mathfrak{H}}=0, \quad l, k=1, \ldots, n .
$$

Combining this with (1.25) and (1.34) and recalling that $\operatorname{Ran} Z \subset \mathfrak{N}^{\perp}, \operatorname{Ran} Z_{2} \subset \mathfrak{N}^{\perp}$, we obtain

$$
\left(\widetilde{\omega}_{l}^{(2)}, \omega_{k}\right)_{\mathfrak{H}}+\left(Z \omega_{l}, Z \omega_{k}\right)_{\mathfrak{H}}+\left(\widetilde{\omega}_{l}, \widetilde{\omega}_{k}\right)_{\mathfrak{H}}+\left(\omega_{l}, \widetilde{\omega}_{k}^{(2)}\right)_{\mathfrak{H}}=0, \quad l, k=1, \ldots, n .
$$

\section{§2. ExPANSIONS FOR THE SPECTRAL PROJECTION $F(t)$}

$$
\text { AND THE OPERATOR } A(t) F(t)
$$

2.1. Expansion for $F(t)$. We consider the spectral projection $F(t)=F(t, \delta)$. By $(1.2)$ and (1.19), for $|t| \leq t^{0}$ we have

$$
F(t)=\sum_{l=1}^{n}\left(\cdot, \varphi_{l}(t)\right)_{\mathfrak{H}} \varphi_{l}(t) .
$$

The operator-valued function $F(t)$ is real-analytic for $|t| \leq t^{0}$. From (1.22) and (1.23) it follows that for $|t| \leq t_{*}$ we have the following convergent power series expansion:

$$
F(t)=P+t F_{1}+t^{2} F_{2}+\cdots .
$$


Here

$$
\begin{aligned}
& F_{1}=\sum_{l=1}^{n}\left(\left(\cdot, \varphi_{l}^{(1)}\right)_{\mathfrak{H}} \omega_{l}+\left(\cdot, \omega_{l}\right)_{\mathfrak{H}} \varphi_{l}^{(1)}\right), \\
& F_{2}=\sum_{l=1}^{n}\left(\left(\cdot, \varphi_{l}^{(2)}\right)_{\mathfrak{H}} \omega_{l}+\left(\cdot, \varphi_{l}^{(1)}\right)_{\mathfrak{H}} \varphi_{l}^{(1)}+\left(\cdot, \omega_{l}\right)_{\mathfrak{H}} \varphi_{l}^{(2)}\right) .
\end{aligned}
$$

Relations (1.25) and (2.2) show that

$$
\begin{aligned}
F_{1} & =\sum_{l=1}^{n}\left(\left(\cdot, Z \omega_{l}\right)_{\mathfrak{H}} \omega_{l}+\left(\cdot, \omega_{l}\right)_{\mathfrak{H}} Z \omega_{l}\right)+Y, \\
Y & =\sum_{l=1}^{n}\left(\left(\cdot, \widetilde{\omega}_{l}\right)_{\mathfrak{H}} \omega_{l}+\left(\cdot, \omega_{l}\right)_{\mathfrak{H}} \widetilde{\omega}_{l}\right) .
\end{aligned}
$$

Clearly, $\left.Y\right|_{\mathfrak{N}^{\perp}}=0$. From (1.36) it follows that $\left(Y \omega_{j}, \omega_{k}\right)_{\mathfrak{H}}=0, j, k=1, \ldots, n$. Hence, $Y=0$. Then (2.4) and (1.23) show that

$$
F_{1}=Z P+P Z^{*}=Z+Z^{*}
$$

(cf. [BSu2, Subsection 2.3]). By (1.7) and (2.6),

$$
\left\|F_{1}\right\| \leq(2 \delta)^{-1 / 2}\left\|X_{1}\right\|
$$

We proceed to calculation of the operator $F_{2}$. By $(2.3),(1.25)$, and (1.34), we have

$$
\begin{aligned}
F_{2}= & \sum_{l=1}^{n}\left(\left(\cdot, Z_{2} \omega_{l}+Z \widetilde{\omega}_{l}+\widetilde{\omega}_{l}^{(2)}\right)_{\mathfrak{H}} \omega_{l}+\left(\cdot, Z \omega_{l}+\widetilde{\omega}_{l}\right)_{\mathfrak{H}}\left(Z \omega_{l}+\widetilde{\omega}_{l}\right)\right) \\
& +\sum_{l=1}^{n}\left(\cdot, \omega_{l}\right)_{\mathfrak{H}}\left(Z_{2} \omega_{l}+Z \widetilde{\omega}_{l}+\widetilde{\omega}_{l}^{(2)}\right)=F_{2}^{(1)}+F_{2}^{(2)}+F_{2}^{(3)}+F_{2}^{(4)}
\end{aligned}
$$

where

$$
\begin{aligned}
F_{2}^{(1)} & =\sum_{l=1}^{n}\left(\left(\cdot, Z_{2} \omega_{l}\right)_{\mathfrak{H}} \omega_{l}+\left(\cdot, \omega_{l}\right)_{\mathfrak{H}} Z_{2} \omega_{l}\right), \\
F_{2}^{(2)} & =\sum_{l=1}^{n}\left(\cdot, Z \omega_{l}\right)_{\mathfrak{H}} Z \omega_{l}, \\
F_{2}^{(3)} & =\sum_{l=1}^{n}\left(\left(\cdot, Z \widetilde{\omega}_{l}\right)_{\mathfrak{H}} \omega_{l}+\left(\cdot, \widetilde{\omega}_{l}\right)_{\mathfrak{H}} Z \omega_{l}+\left(\cdot, Z \omega_{l}\right)_{\mathfrak{H}} \widetilde{\omega}_{l}+\left(\cdot, \omega_{l}\right)_{\mathfrak{H}} Z \widetilde{\omega}_{l}\right), \\
F_{2}^{(4)} & =\sum_{l=1}^{n}\left(\left(\cdot, \widetilde{\omega}_{l}^{(2)}\right)_{\mathfrak{H}} \omega_{l}+\left(\cdot, \widetilde{\omega}_{l}\right)_{\mathfrak{H}} \widetilde{\omega}_{l}+\left(\cdot, \omega_{l}\right)_{\mathfrak{H}} \widetilde{\omega}_{l}^{(2)}\right) .
\end{aligned}
$$

Taking (1.23) and the identities $Z P=Z, Z_{2} P=Z_{2}, P Z_{2}^{*}=Z_{2}^{*}$ into account, we obtain

$$
\begin{aligned}
& F_{2}^{(1)}=Z_{2} P+P Z_{2}^{*}=Z_{2}+Z_{2}^{*}, \\
& F_{2}^{(2)}=Z P Z^{*}=Z Z^{*} .
\end{aligned}
$$

Next, from (2.5) and the identity $Y=0$ we deduce that

$$
F_{2}^{(3)}=Y Z^{*}+Z Y=0 .
$$


Consider the term $F_{2}^{(4)}$. Clearly, $\left.F_{2}^{(4)}\right|_{\mathfrak{N}^{\perp}}=0$. By (1.36), (1.23), and (1.37), we have

$$
\begin{aligned}
\left(F_{2}^{(4)} \omega_{j}, \omega_{k}\right)_{\mathfrak{H}} & =\sum_{l=1}^{n}\left(\left(\omega_{j}, \widetilde{\omega}_{l}^{(2)}\right) \delta_{l k}+\left(\omega_{j}, \widetilde{\omega}_{l}\right)\left(\widetilde{\omega}_{l}, \omega_{k}\right)+\delta_{j l}\left(\widetilde{\omega}_{l}^{(2)}, \omega_{k}\right)\right) \\
& =\left(\omega_{j}, \widetilde{\omega}_{k}^{(2)}\right)+\sum_{l=1}^{n}\left(\widetilde{\omega}_{j}, \omega_{l}\right)\left(\omega_{l}, \widetilde{\omega}_{k}\right)+\left(\widetilde{\omega}_{j}^{(2)}, \omega_{k}\right) \\
& =\left(\omega_{j}, \widetilde{\omega}_{k}^{(2)}\right)+\left(\widetilde{\omega}_{j}, \widetilde{\omega}_{k}\right)+\left(\widetilde{\omega}_{j}^{(2)}, \omega_{k}\right)=-\left(Z \omega_{j}, Z \omega_{k}\right)
\end{aligned}
$$

for $j, k=1, \ldots, n$. Hence,

$$
F_{2}^{(4)}=-Z^{*} Z
$$

Finally, relations (2.8)-(2.12) imply the representation

$$
F_{2}=Z_{2}+Z_{2}^{*}+Z Z^{*}-Z^{*} Z \text {. }
$$

By (1.7) and (1.16),

$$
\left\|F_{2}\right\| \leq 2\left\|Z_{2}\right\|+2\|Z\|^{2} \leq 3(4 \delta)^{-1}\left\|X_{1}\right\|^{2} .
$$

2.2. Expansion for $A(t) F(t)$. In accordance with (1.2) and (1.19), for $|t| \leq t^{0}$ we have

$$
A(t) F(t)=\sum_{l=1}^{n} \lambda_{l}(t)\left(\cdot, \varphi_{l}(t)\right)_{\mathfrak{H}} \varphi_{l}(t) .
$$

The operator-valued function (2.15) is real-analytic for $|t| \leq t^{0}$. From (1.21), (1.22), and (1.30) it follows that for $|t| \leq t_{*}$ we have the following convergent power series expansion:

$$
A(t) F(t)=t^{2} S P+t^{3} K+t^{4} W+\cdots .
$$

Here

$$
\begin{aligned}
K= & \sum_{l=1}^{n} \gamma_{l}\left(\left(\cdot, \omega_{l}\right)_{\mathfrak{H}} \varphi_{l}^{(1)}+\left(\cdot, \varphi_{l}^{(1)}\right)_{\mathfrak{H}} \omega_{l}\right)+\sum_{l=1}^{n} \mu_{l}\left(\cdot, \omega_{l}\right)_{\mathfrak{H}} \omega_{l}, \\
W= & \sum_{l=1}^{n} \gamma_{l}\left(\left(\cdot, \omega_{l}\right)_{\mathfrak{H}} \varphi_{l}^{(2)}+\left(\cdot, \varphi_{l}^{(1)}\right)_{\mathfrak{H}} \varphi_{l}^{(1)}+\left(\cdot, \varphi_{l}^{(2)}\right)_{\mathfrak{H}} \omega_{l}\right) \\
& +\sum_{l=1}^{n} \mu_{l}\left(\left(\cdot, \omega_{l}\right)_{\mathfrak{H}} \varphi_{l}^{(1)}+\left(\cdot, \varphi_{l}^{(1)}\right)_{\mathfrak{H}} \omega_{l}\right)+\sum_{l=1}^{n} \nu_{l}\left(\cdot, \omega_{l}\right)_{\mathfrak{H}} \omega_{l} .
\end{aligned}
$$

From (2.17) and (1.25) we obtain (cf. [BSu2, Subsection 4.2])

$$
\begin{aligned}
K & =K_{0}+N, \\
K_{0} & =\sum_{l=1}^{n} \gamma_{l}\left(\left(\cdot, \omega_{l}\right)_{\mathfrak{H}} Z \omega_{l}+\left(\cdot, Z \omega_{l}\right)_{\mathfrak{H}} \omega_{l}\right), \\
N & =\sum_{l=1}^{n} \gamma_{l}\left(\left(\cdot, \omega_{l}\right)_{\mathfrak{H}} \widetilde{\omega}_{l}+\left(\cdot, \widetilde{\omega}_{l}\right)_{\mathfrak{H}} \omega_{l}\right)+\sum_{l=1}^{n} \mu_{l}\left(\cdot, \omega_{l}\right)_{\mathfrak{H}} \omega_{l} .
\end{aligned}
$$

In the orthogonal decomposition $\mathfrak{H}=\mathfrak{N} \oplus \mathfrak{N}^{\perp}$, the operator $K_{0}$ acts "crosswise": it takes $\mathfrak{N}$ into $\mathfrak{N}^{\perp}$ and $\mathfrak{N}^{\perp}$ into $\mathfrak{N}$. On the contrary, the operator $N$ is reduced by this decomposition: it takes $\mathfrak{N}$ into $\mathfrak{N}$, and $\left.N\right|_{\mathfrak{N}^{\perp}}=0$.

Relations (2.20) and (1.30) directly imply the formula

$$
K_{0}=Z S P+S P Z^{*}
$$

(cf. BSu2, Subsection 4.2]). From (2.22), (1.7), and (1.11) it follows that

$$
\left\|K_{0}\right\| \leq(2 \delta)^{-1 / 2}\left\|X_{1}\right\|^{3} .
$$


The operator $N$ was calculated in BSu2, Subsection 4.3]. We repeat the arguments for completeness. We calculate the matrix entries of the operator (2.21):

$$
\left(N \omega_{j}, \omega_{k}\right)_{\mathfrak{H}}=\gamma_{j}\left(\widetilde{\omega}_{j}, \omega_{k}\right)_{\mathfrak{H}}+\gamma_{k}\left(\omega_{j}, \widetilde{\omega}_{k}\right)_{\mathfrak{H}}+\mu_{j} \delta_{j k}, \quad j, k=1, \ldots, n .
$$

The further calculation is based on the identity

$$
\left(A(t) \varphi_{j}(t), \varphi_{k}(t)\right)_{\mathfrak{H}}=\lambda_{j}(t) \delta_{j k}, \quad j, k=1, \ldots, n .
$$

The left-hand side of $(2.25)$ can be written as $\left(\left(X_{0}+t X_{1}\right) \varphi_{j}(t),\left(X_{0}+t X_{1}\right) \varphi_{k}(t)\right)_{\mathfrak{H}_{*}}$. We substitute expansions (1.21) and (1.22) in (2.25) and compare the coefficients of $t^{3}$. Since $X_{0} \omega_{j}=X_{0} \omega_{k}=0$, we arrive at the identity

$$
\left(X_{0} \varphi_{j}^{(1)}+X_{1} \omega_{j}, X_{0} \varphi_{k}^{(2)}+X_{1} \varphi_{k}^{(1)}\right)_{\mathfrak{H}_{*}}+\left(X_{0} \varphi_{j}^{(2)}+X_{1} \varphi_{j}^{(1)}, X_{0} \varphi_{k}^{(1)}+X_{1} \omega_{k}\right)_{\mathfrak{H}_{*}}=\mu_{j} \delta_{j k} .
$$

Combining this with (1.26) and recalling that $X_{0}^{*} R=0$, we obtain

$$
\left(R \omega_{j}, X_{1} \varphi_{k}^{(1)}\right)_{\mathfrak{H}_{*}}+\left(X_{1} \varphi_{j}^{(1)}, R \omega_{k}\right)_{\mathfrak{H}_{*}}=\mu_{j} \delta_{j k} .
$$

Using (1.25) and (1.28), we see that identity (2.26) takes the form

$$
\left(R \omega_{j}, X_{1} Z \omega_{k}\right)_{\mathfrak{H}_{*}}+\left(X_{1} Z \omega_{j}, R \omega_{k}\right)_{\mathfrak{H}_{*}}+\gamma_{j}\left(\omega_{j}, \widetilde{\omega}_{k}\right)_{\mathfrak{H}}+\gamma_{k}\left(\widetilde{\omega}_{j}, \omega_{k}\right)_{\mathfrak{H}}=\mu_{j} \delta_{j k} .
$$

Finally, using (1.36) and (2.24), from (2.27) we obtain

$$
\left(N \omega_{j}, \omega_{k}\right)_{\mathfrak{H}}=\left(R \omega_{j}, X_{1} Z \omega_{k}\right)_{\mathfrak{H}_{*}}+\left(X_{1} Z \omega_{j}, R \omega_{k}\right)_{\mathfrak{H}_{*}}, \quad j, k=1, \ldots, n .
$$

Thus,

$$
N=Z^{*} X_{1}^{*} R P+(R P)^{*} X_{1} Z .
$$

From (1.7), (1.9), and (2.28) it follows that

$$
\|N\| \leq(2 \delta)^{-1 / 2}\left\|X_{1}\right\|^{3} .
$$

Now, relations (2.23) and (2.29) imply the following estimate for the operator (2.19):

$$
\|K\| \leq 2(2 \delta)^{-1 / 2}\left\|X_{1}\right\|^{3} .
$$

2.3. Calculation of the operator $W$. Substituting (1.25) and (1.34) in (2.18), we see that

$$
W=W_{0}+K_{1}+N_{1}
$$

where

$$
\begin{aligned}
W_{0}= & \sum_{l=1}^{n} \gamma_{l}\left(\cdot, Z \omega_{l}\right)_{\mathfrak{H}} Z \omega_{l} \\
K_{1}= & \sum_{l=1}^{n} \gamma_{l}\left(\left(\cdot, \omega_{l}\right)_{\mathfrak{H}}\left(Z_{2} \omega_{l}+Z \widetilde{\omega}_{l}\right)+\left(\cdot, Z_{2} \omega_{l}+Z \widetilde{\omega}_{l}\right)_{\mathfrak{H}} \omega_{l}\right) \\
& +\sum_{l=1}^{n} \gamma_{l}\left(\left(\cdot, \widetilde{\omega}_{l}\right)_{\mathfrak{H}} Z \omega_{l}+\left(\cdot, Z \omega_{l}\right)_{\mathfrak{H}} \widetilde{\omega}_{l}\right)+\sum_{l=1}^{n} \mu_{l}\left(\left(\cdot, \omega_{l}\right)_{\mathfrak{H}} Z \omega_{l}+\left(\cdot, Z \omega_{l}\right)_{\mathfrak{H}} \omega_{l}\right), \\
N_{1}= & \sum_{l=1}^{n} \gamma_{l}\left(\left(\cdot, \omega_{l}\right)_{\mathfrak{H}} \widetilde{\omega}_{l}^{(2)}+\left(\cdot, \widetilde{\omega}_{l}^{(2)}\right)_{\mathfrak{H}} \omega_{l}+\left(\cdot, \widetilde{\omega}_{l}\right)_{\mathfrak{H}} \widetilde{\omega}_{l}\right) \\
+ & \sum_{l=1}^{n} \mu_{l}\left(\left(\cdot, \omega_{l}\right)_{\mathfrak{H}} \widetilde{\omega}_{l}+\left(\cdot, \widetilde{\omega}_{l}\right)_{\mathfrak{H}} \omega_{l}\right)+\sum_{l=1}^{n} \nu_{l}\left(\cdot, \omega_{l}\right)_{\mathfrak{H}} \omega_{l} .
\end{aligned}
$$


In the orthogonal decomposition $\mathfrak{H}=\mathfrak{N} \oplus \mathfrak{N}^{\perp}$, the operator $K_{1}$ acts "crosswise": it takes $\mathfrak{N}$ into $\mathfrak{N}^{\perp}$ and $\mathfrak{N}^{\perp}$ into $\mathfrak{N}$. The operators $W_{0}$ and $N_{1}$ are reduced by this decomposition: $W_{0}$ takes $\mathfrak{N}^{\perp}$ into itself, and $\left.W_{0}\right|_{\mathfrak{N}}=0$; the operator $N_{1}$ takes $\mathfrak{N}$ into itself, and $\left.N_{1}\right|_{\mathfrak{N}^{\perp}}=0$.

By (1.30), the operator (2.32) takes the form

$$
W_{0}=Z S Z^{*} \text {. }
$$

From (1.7) and (1.11) it follows that the operator (2.35) satisfies the estimate

$$
\left\|W_{0}\right\| \leq(8 \delta)^{-1}\left\|X_{1}\right\|^{4} .
$$

By (1.30) and (2.21), the operator (2.33) admits the following representation:

$$
K_{1}=Z_{2} S P+S P Z_{2}^{*}+Z N+N Z^{*} .
$$

Relations (1.7), (1.11), (1.16), and (2.29) imply the inequality

$$
\left\|K_{1}\right\| \leq \delta^{-1}\left\|X_{1}\right\|^{4} \text {. }
$$

Now we consider the operator (2.34). We calculate its matrix entries:

$$
\begin{aligned}
\left(N_{1} \omega_{j}, \omega_{k}\right)_{\mathfrak{H}}= & \gamma_{j}\left(\widetilde{\omega}_{j}^{(2)}, \omega_{k}\right)_{\mathfrak{H}}+\gamma_{k}\left(\omega_{j}, \widetilde{\omega}_{k}^{(2)}\right)_{\mathfrak{H}}+\sum_{l=1}^{n} \gamma_{l}\left(\omega_{j}, \widetilde{\omega}_{l}\right)_{\mathfrak{H}}\left(\widetilde{\omega}_{l}, \omega_{k}\right)_{\mathfrak{H}} \\
& +\mu_{j}\left(\widetilde{\omega}_{j}, \omega_{k}\right)_{\mathfrak{H}}+\mu_{k}\left(\omega_{j}, \widetilde{\omega}_{k}\right)_{\mathfrak{H}}+\nu_{j} \delta_{j k}, \quad j, k=1, \ldots, n .
\end{aligned}
$$

By (1.36) and (1.30), we have

$$
\sum_{l=1}^{n} \gamma_{l}\left(\omega_{j}, \widetilde{\omega}_{l}\right)_{\mathfrak{H}}\left(\widetilde{\omega}_{l}, \omega_{k}\right)_{\mathfrak{H}}=\sum_{l=1}^{n} \gamma_{l}\left(\widetilde{\omega}_{j}, \omega_{l}\right)_{\mathfrak{H}}\left(\omega_{l}, \widetilde{\omega}_{k}\right)_{\mathfrak{H}}=\left(S \widetilde{\omega}_{j}, \widetilde{\omega}_{k}\right)_{\mathfrak{H}}, \quad j, k=1, \ldots, n .
$$

Hence,

$$
\begin{aligned}
\left(N_{1} \omega_{j}, \omega_{k}\right)_{\mathfrak{H}}= & \gamma_{j}\left(\widetilde{\omega}_{j}^{(2)}, \omega_{k}\right)_{\mathfrak{H}}+\gamma_{k}\left(\omega_{j}, \widetilde{\omega}_{k}^{(2)}\right)_{\mathfrak{H}}+\left(S \widetilde{\omega}_{j}, \widetilde{\omega}_{k}\right)_{\mathfrak{H}} \\
& +\mu_{j}\left(\widetilde{\omega}_{j}, \omega_{k}\right)_{\mathfrak{H}}+\mu_{k}\left(\omega_{j}, \widetilde{\omega}_{k}\right)_{\mathfrak{H}}+\nu_{j} \delta_{j k}, \quad j, k=1, \ldots, n .
\end{aligned}
$$

For further calculations, we use (2.25) once again. Comparing the coefficients of $t^{4}$ in (2.25), we obtain

$$
\begin{aligned}
\left(X_{0} \varphi_{j}^{(1)}\right. & \left.+X_{1} \omega_{j}, X_{0} \varphi_{k}^{(3)}+X_{1} \varphi_{k}^{(2)}\right)_{\mathfrak{H}_{*}}+\left(X_{0} \varphi_{j}^{(2)}+X_{1} \varphi_{j}^{(1)}, X_{0} \varphi_{k}^{(2)}+X_{1} \varphi_{k}^{(1)}\right)_{\mathfrak{H}_{*}} \\
& +\left(X_{0} \varphi_{j}^{(3)}+X_{1} \varphi_{j}^{(2)}, X_{0} \varphi_{k}^{(1)}+X_{1} \omega_{k}\right)_{\mathfrak{H}_{*}}=\nu_{j} \delta_{j k}, \quad j, k=1, \ldots, n .
\end{aligned}
$$

Using (1.26) and (1.35) and taking the identity $X_{0}^{*} R=0$ into account, we rewrite (2.40) as

$$
\left(R \omega_{j}, X_{1} \varphi_{k}^{(2)}\right)_{\mathfrak{H}_{*}}+\left(R_{2} \omega_{j}+R \widetilde{\omega}_{j}, R_{2} \omega_{k}+R \widetilde{\omega}_{k}\right)_{\mathfrak{H}_{*}}+\left(X_{1} \varphi_{j}^{(2)}, R \omega_{k}\right)_{\mathfrak{H}_{*}}=\nu_{j} \delta_{j k}
$$

From (1.17) and the identity $X_{0}^{*} R=0$ it follows that

$$
\left(R \widetilde{\omega}_{j}, R_{2} \omega_{k}\right)_{\mathfrak{H}_{*}}+\left(R_{2} \omega_{j}, R \widetilde{\omega}_{k}\right)_{\mathfrak{H}_{*}}=\left(R \widetilde{\omega}_{j}, X_{1} Z \omega_{k}\right)_{\mathfrak{H}_{*}}+\left(X_{1} Z \omega_{j}, R \widetilde{\omega}_{k}\right)_{\mathfrak{H}_{*}} .
$$

By (1.34), (1.10), and (2.42), relation (2.41) turns into

$$
\begin{aligned}
& \left(R \omega_{j}, X_{1}\left(Z_{2} \omega_{k}+Z \widetilde{\omega}_{k}+\widetilde{\omega}_{k}^{(2)}\right)\right)_{\mathfrak{H}_{*}}+\left(X_{1}\left(Z_{2} \omega_{j}+Z \widetilde{\omega}_{j}+\widetilde{\omega}_{j}^{(2)}\right), R \omega_{k}\right)_{\mathfrak{H}_{*}} \\
& \quad+\left(R_{2}^{*} R_{2} \omega_{j}, \omega_{k}\right)_{\mathfrak{H}}+\left(S \widetilde{\omega}_{j}, \widetilde{\omega}_{k}\right)_{\mathfrak{H}}+\left(R \widetilde{\omega}_{j}, X_{1} Z \omega_{k}\right)_{\mathfrak{H}_{*}}+\left(X_{1} Z \omega_{j}, R \widetilde{\omega}_{k}\right)_{\mathfrak{H}_{*}}=\nu_{j} \delta_{j k} .
\end{aligned}
$$

Next, by (2.28), we have

$$
\begin{array}{r}
\left(R \omega_{j}, X_{1} Z \widetilde{\omega}_{k}\right)_{\mathfrak{H}_{*}}+\left(X_{1} Z \widetilde{\omega}_{j}, R \omega_{k}\right)_{\mathfrak{H}_{*}}+\left(R \widetilde{\omega}_{j}, X_{1} Z \omega_{k}\right)_{\mathfrak{H}_{*}}+\left(X_{1} Z \omega_{j}, R \widetilde{\omega}_{k}\right)_{\mathfrak{H}_{*}} \\
=\left(N \omega_{j}, \widetilde{\omega}_{k}\right)_{\mathfrak{H}}+\left(N \widetilde{\omega}_{j}, \omega_{k}\right)_{\mathfrak{H}} .
\end{array}
$$


Then $(2.43)$ can be written as

$$
\begin{aligned}
& \left(R \omega_{j}, X_{1} Z_{2} \omega_{k}\right)_{\mathfrak{H}_{*}}+\left(X_{1} Z_{2} \omega_{j}, R \omega_{k}\right)_{\mathfrak{H}_{*}}+\left(R_{2}^{*} R_{2} \omega_{j}, \omega_{k}\right)_{\mathfrak{H}}+\left(S \widetilde{\omega}_{j}, \widetilde{\omega}_{k}\right)_{\mathfrak{H}} \\
& \quad+\left(N \omega_{j}, \widetilde{\omega}_{k}\right)_{\mathfrak{H}}+\left(N \widetilde{\omega}_{j}, \omega_{k}\right)_{\mathfrak{H}}+\left(R \omega_{j}, X_{1} \widetilde{\omega}_{k}^{(2)}\right)_{\mathfrak{H} *}+\left(X_{1} \widetilde{\omega}_{j}^{(2)}, R \omega_{k}\right)_{\mathfrak{H} *}=\nu_{j} \delta_{j k} .
\end{aligned}
$$

We transform the sum of the last two terms on the left with the help of (1.28) and (1.37):

$$
\begin{aligned}
& \left(R \omega_{j}, X_{1} \widetilde{\omega}_{k}^{(2)}\right)_{\mathfrak{H}_{*}}+\left(X_{1} \widetilde{\omega}_{j}^{(2)}, R \omega_{k}\right)_{\mathfrak{H}_{*}}=\gamma_{j}\left(\omega_{j}, \widetilde{\omega}_{k}^{(2)}\right)_{\mathfrak{H}}+\gamma_{k}\left(\widetilde{\omega}_{j}^{(2)}, \omega_{k}\right)_{\mathfrak{H}} \\
& =-\gamma_{j}\left(\widetilde{\omega}_{j}^{(2)}, \omega_{k}\right)_{\mathfrak{H}}-\gamma_{k}\left(\omega_{j}, \widetilde{\omega}_{k}^{(2)}\right)_{\mathfrak{H}}-\left(\gamma_{j}+\gamma_{k}\right)\left(\widetilde{\omega}_{j}, \widetilde{\omega}_{k}\right)_{\mathfrak{H}}-\left(\gamma_{j}+\gamma_{k}\right)\left(Z \omega_{j}, Z \omega_{k}\right)_{\mathfrak{H}} .
\end{aligned}
$$

Now we transform the terms containing $N$ in (2.44) with the help of (1.23), (2.24), (1.36), and (1.30):

$$
\begin{aligned}
& \left(N \omega_{j}, \widetilde{\omega}_{k}\right)_{\mathfrak{H}}+\left(N \widetilde{\omega}_{j}, \omega_{k}\right)_{\mathfrak{H}} \\
& =\sum_{l=1}^{n}\left(\left(N \omega_{j}, \omega_{l}\right)_{\mathfrak{H}}\left(\omega_{l}, \widetilde{\omega}_{k}\right)_{\mathfrak{H}}+\left(\widetilde{\omega}_{j}, \omega_{l}\right)_{\mathfrak{H}}\left(\omega_{l}, N \omega_{k}\right)_{\mathfrak{H}}\right) \\
& =\sum_{l=1}^{n}\left(\gamma_{j}\left(\widetilde{\omega}_{j}, \omega_{l}\right)_{\mathfrak{H}}+\gamma_{l}\left(\omega_{j}, \widetilde{\omega}_{l}\right)_{\mathfrak{H}}+\mu_{j} \delta_{j l}\right)\left(\omega_{l}, \widetilde{\omega}_{k}\right)_{\mathfrak{H}} \\
& \quad+\sum_{l=1}^{n}\left(\widetilde{\omega}_{j}, \omega_{l}\right)_{\mathfrak{H}}\left(\gamma_{l}\left(\widetilde{\omega}_{l}, \omega_{k}\right)_{\mathfrak{H}}+\gamma_{k}\left(\omega_{l}, \widetilde{\omega}_{k}\right)_{\mathfrak{H}}+\mu_{l} \delta_{l k}\right) \\
& =\mu_{j}\left(\omega_{j}, \widetilde{\omega}_{k}\right)_{\mathfrak{H}}+\mu_{k}\left(\widetilde{\omega}_{j}, \omega_{k}\right)_{\mathfrak{H}}+\sum_{l=1}^{n}\left(\gamma_{j}+\gamma_{k}-2 \gamma_{l}\right)\left(\widetilde{\omega}_{j}, \omega_{l}\right)_{\mathfrak{H}}\left(\omega_{l}, \widetilde{\omega}_{k}\right)_{\mathfrak{H}} \\
& =-\mu_{j}\left(\widetilde{\omega}_{j}, \omega_{k}\right)_{\mathfrak{H}}-\mu_{k}\left(\omega_{j}, \widetilde{\omega}_{k}\right)_{\mathfrak{H}}+\left(\gamma_{j}+\gamma_{k}\right)\left(\widetilde{\omega}_{j}, \widetilde{\omega}_{k}\right)_{\mathfrak{H}}-2\left(S \widetilde{\omega}_{j}, \widetilde{\omega}_{k}\right)_{\mathfrak{H}} .
\end{aligned}
$$

As a result, we see that formulas (2.44)-(2.46) imply the relation

$$
\begin{aligned}
\left(R \omega_{j}\right. & \left., X_{1} Z_{2} \omega_{k}\right)_{\mathfrak{H}_{*}}+\left(X_{1} Z_{2} \omega_{j}, R \omega_{k}\right)_{\mathfrak{H}_{*}}+\left(R_{2}^{*} R_{2} \omega_{j}, \omega_{k}\right)_{\mathfrak{H}}-\mu_{j}\left(\widetilde{\omega}_{j}, \omega_{k}\right)_{\mathfrak{H}} \\
& -\mu_{k}\left(\omega_{j}, \widetilde{\omega}_{k}\right)_{\mathfrak{H}}-\gamma_{j}\left(\widetilde{\omega}_{j}^{(2)}, \omega_{k}\right)_{\mathfrak{H}}-\gamma_{k}\left(\omega_{j}, \widetilde{\omega}_{k}^{(2)}\right)_{\mathfrak{H}}-\left(S \widetilde{\omega}_{j}, \widetilde{\omega}_{k}\right)_{\mathfrak{H}} \\
& -\left(\gamma_{j}+\gamma_{k}\right)\left(Z \omega_{j}, Z \omega_{k}\right)_{\mathfrak{H}}=\nu_{j} \delta_{j k} .
\end{aligned}
$$

Comparing this with (2.39), we arrive at the formula

$$
\begin{aligned}
& \left(N_{1} \omega_{j}, \omega_{k}\right)_{\mathfrak{H}}=\left(R \omega_{j}, X_{1} Z_{2} \omega_{k}\right)_{\mathfrak{H}_{*}}+\left(X_{1} Z_{2} \omega_{j}, R \omega_{k}\right)_{\mathfrak{H}_{*}} \\
& \quad+\left(R_{2}^{*} R_{2} \omega_{j}, \omega_{k}\right)_{\mathfrak{H}}-\left(\gamma_{j}+\gamma_{k}\right)\left(Z \omega_{j}, Z \omega_{k}\right)_{\mathfrak{H}}, \quad j, k=1, \ldots, n .
\end{aligned}
$$

We transform the last term on the right-hand side of (2.47), taking (1.29) into account:

$$
\begin{aligned}
\left(\gamma_{j}+\gamma_{k}\right)\left(Z \omega_{j}, Z \omega_{k}\right)_{\mathfrak{H}} & =\gamma_{j}\left(\omega_{j}, Z^{*} Z \omega_{k}\right)_{\mathfrak{H}}+\gamma_{k}\left(Z^{*} Z \omega_{j}, \omega_{k}\right)_{\mathfrak{H}} \\
& =\left(S \omega_{j}, Z^{*} Z \omega_{k}\right)_{\mathfrak{H}}+\left(Z^{*} Z \omega_{j}, S \omega_{k}\right)_{\mathfrak{H}}
\end{aligned}
$$

Then from (2.47) it follows that

$$
N_{1}=N_{1}^{0}-Z^{*} Z S P-S P Z^{*} Z,
$$

where

$$
N_{1}^{0}=Z_{2}^{*} X_{1}^{*} R P+(R P)^{*} X_{1} Z_{2}+R_{2}^{*} R_{2} P .
$$

Relations (1.7), (1.9), (1.11), (1.16), and (1.18) imply the following estimates for the operators (2.49) and (2.48):

$$
\left\|N_{1}^{0}\right\| \leq 9(8 \delta)^{-1}\left\|X_{1}\right\|^{4}, \quad\left\|N_{1}\right\| \leq 11(8 \delta)^{-1}\left\|X_{1}\right\|^{4} .
$$

The next estimate for the operator (2.31) is a consequence of (2.36), (2.38), and (2.50):

$$
\|W\| \leq 5(2 \delta)^{-1}\left\|X_{1}\right\|^{4} .
$$


2.4. The convergent power series expansions (2.1) and (2.16) are not quite suitable for our purposes. We only need estimates for the error terms

$$
\begin{aligned}
F_{3}(t) & =F(t)-P-t F_{1}-t^{2} F_{2}, \\
\Psi(t) & =A(t) F(t)-t^{2} S P-t^{3} K-t^{4} W,
\end{aligned}
$$

but on a wider interval $|t| \leq t^{0}$ and with constants controlled explicitly. Such estimates can be obtained by integration of the difference of resolvents of $A(t)$ and $A_{0}$ over a suitable contour in the complex plane (cf. [BSu1, Chapter 1, $\S \S 2-4]$, BSu2, $\S \S 2-4]$ ). Below in $\S 3$, an approximation for the difference of resolvents on the contour is obtained, and $\S 4$ contains estimates for the operators (2.52) and (2.53).

\section{§3. Approximation for the Difference of Resolvents ON THE CONTOUR $\Gamma_{\delta}$}

3.1. The contour $\Gamma_{\delta}$. Let $\Gamma_{\delta} \subset \mathbb{C}$ be the contour that envelopes the real interval $[0, \delta]$ equidistantly at the distance $\delta$. We denote

$$
R_{z}(t)=(A(t)-z I)^{-1}, \quad R_{z}(0)=\left(A_{0}-z I\right)^{-1},
$$

where $z$ is a common regular point of $A(t)$ and $A_{0}$.

By (1.2), for $|t| \leq t^{0}$ the distance from $\Gamma_{\delta}$ to the spectrum of $A(t)$ is at least $\delta$, whence

$$
\left\|R_{z}(t)\right\| \leq \delta^{-1}, \quad z \in \Gamma_{\delta}, \quad|t| \leq t^{0} .
$$

Below we use the notation

$$
\Omega_{z}(t)=I+(z+2 \delta) R_{z}(t) .
$$

Since $|z| \leq 2 \delta$ for $z \in \Gamma_{\delta}$, inequality (3.1) implies

$$
\left\|\Omega_{z}(t)\right\| \leq 5, \quad z \in \Gamma_{\delta}, \quad|t| \leq t^{0} .
$$

3.2. The resolvent identity. The usual resolvent identity is not applicable now. We use its analog for the operators defined in terms of quadratic forms with common domain; see BSu1, Chapter 1, $\S 2$ ]. To describe the corresponding identity, we define the metric form $\left\|X_{0} u\right\|_{\mathfrak{H}_{*}}^{2}+2 \delta\|u\|_{\mathfrak{H}}^{2}$ on $\operatorname{Dom} X_{0}$. Then Dom $X_{0}$ converts into the Hilbert space $\mathfrak{d}$. The form

$$
a(t)[u, u]-a(0)[u, u]=2 t \operatorname{Re}\left(X_{0} u, X_{1} u\right)_{\mathfrak{H}_{*}}+t^{2}\left\|X_{1} u\right\|_{\mathfrak{H}_{*}}^{2}, \quad u \in \mathfrak{d},
$$

generates a continuous operator $T_{\delta}(t)$ in $\mathfrak{d}$. The operator $T_{\delta}(t)$ is represented as

$$
T_{\delta}(t)=t T_{\delta}^{(1)}+t^{2} T_{\delta}^{(2)},
$$

where $T_{\delta}^{(1)}$ corresponds to the form $2 \operatorname{Re}\left(X_{0} u, X_{1} u\right)_{\mathfrak{H}_{*}}$, and $T_{\delta}^{(2)}$ corresponds to the form $\left\|X_{1} u\right\|_{\mathfrak{H}_{*}}^{2}$. As was shown in [BSu1, (3.7)-(3.9)], we have

$$
\begin{aligned}
\left\|T_{\delta}^{(1)}\right\|_{\mathfrak{o} \rightarrow \mathfrak{d}} & \leq(2 \delta)^{-1 / 2}\left\|X_{1}\right\|, \\
\left\|T_{\delta}^{(2)}\right\|_{\mathfrak{d} \rightarrow \mathfrak{d}} & \leq(2 \delta)^{-1}\left\|X_{1}\right\|^{2}, \\
\left\|T_{\delta}(t)\right\|_{\mathfrak{d} \rightarrow \mathfrak{d}} & \leq \frac{\sqrt{2}+1}{2}|t| \delta^{-1 / 2}\left\|X_{1}\right\|, \quad|t| \leq t^{0} .
\end{aligned}
$$

By BSu1, Chapter 1, (3.11)],

$$
R_{z}(0)-R_{z}(t)=\Omega_{z}(0) T_{\delta}(t) R_{z}(t), \quad z \in \Gamma_{\delta} .
$$

We shall also need the following estimates proved in [BSu1, Chapter 1, (2.15), (2.16)]:

$$
\begin{aligned}
\left\|L R_{z}(0)\right\|_{\mathfrak{H} \rightarrow \mathfrak{H}} & \leq(2 \delta)^{-1}\|L\|_{\mathfrak{d} \rightarrow \mathfrak{d}}\left\|\Omega_{z}(0)\right\|_{\mathfrak{H} \rightarrow \mathfrak{H}}, \\
\left\|L R_{z}(t)\right\|_{\mathfrak{H} \rightarrow \mathfrak{H}} & \leq \sqrt{2}(2 \delta)^{-1}\|L\|_{\mathfrak{d} \rightarrow \mathfrak{o}}\left\|\Omega_{z}(t)\right\|_{\mathfrak{H} \rightarrow \mathfrak{H}},
\end{aligned}
$$


where $L$ is a continuous operator in $\mathfrak{d}$. Taking (3.2) into account and using induction, we easily see that

$$
\begin{aligned}
& \left\|L_{1} \Omega_{z}(0) \ldots L_{p} \Omega_{z}(0) L_{p+1} R_{z}(t)\right\|_{\mathfrak{H} \rightarrow \mathfrak{H}} \\
& \quad \leq \sqrt{2}(2 \delta)^{-1}\left\|\Omega_{z}(t)\right\|_{\mathfrak{H} \rightarrow \mathfrak{H}}\left(1+\frac{|z+2 \delta|}{2 \delta}\left\|\Omega_{z}(0)\right\|_{\mathfrak{H} \rightarrow \mathfrak{H}}\right)^{p} \prod_{j=1}^{p+1}\left\|L_{j}\right\|_{\mathfrak{d} \rightarrow \mathfrak{d}},
\end{aligned}
$$

where $p \in \mathbb{Z}_{+}$, and $L_{1}, \ldots, L_{p+1}$ are continuous operators in $\mathfrak{d}$. (Cf. BSu1, Chapter 1, $(2.17)]$.

Combining (3.9), (3.3), and the estimate $|z| \leq 2 \delta$ for $z \in \Gamma_{\delta}$, we obtain

$$
\begin{array}{r}
\left\|L_{1} \Omega_{z}(0) \ldots L_{p} \Omega_{z}(0) L_{p+1} R_{z}(t)\right\|_{\mathfrak{H} \rightarrow \mathfrak{H}} \leq 5 \sqrt{2}(2 \delta)^{-1} 11^{p} \prod_{j=1}^{p+1}\left\|L_{j}\right\|_{\mathfrak{d} \rightarrow \mathfrak{d}}, \\
z \in \Gamma_{\delta}, \quad|t| \leq t^{0} .
\end{array}
$$

3.3. The difference of resolvents. Separation of the main part. In BSu1, Chapter $1, \S 3]$, the terms up to the order of $t^{2}$ were separated from the difference of resolvents of $A(t)$ and $A(0)$, while for the error term an estimate of order $t^{3}$ was proved. In $\mathrm{BSu} 2$, $\S 3]$, the terms up to those of order $t^{3}$ were separated, while for the error term an estimate of order $t^{4}$ was obtained. Now we need two representations for the operator $R_{z}(0)-R_{z}(t)$, one with the error term of order $O\left(t^{3}\right)$ (deduction of this representation given below is borrowed from [BSu1]), and the second with the error term of order $O\left(t^{5}\right)$ (it is obtained below in Subsection 3.4).

We shall iterate identity (3.8). By (3.4), we have

$$
\begin{aligned}
R_{z}(0)-R_{z}(t)= & \Omega_{z}(0)\left(t T_{\delta}^{(1)}+t^{2} T_{\delta}^{(2)}\right)\left(R_{z}(0)-\Omega_{z}(0) T_{\delta}(t) R_{z}(t)\right) \\
=t I_{z}^{(1)} & -t \Omega_{z}(0) T_{\delta}^{(1)} \Omega_{z}(0)\left(t T_{\delta}^{(1)}+t^{2} T_{\delta}^{(2)}\right) R_{z}(t) \\
& +t^{2} \Omega_{z}(0) T_{\delta}^{(2)} R_{z}(t)
\end{aligned}
$$

where the term $I_{z}^{(1)}=\Omega_{z}(0) T_{\delta}^{(1)} R_{z}(0)$ does not depend on $t$. Iterating once again, we obtain

$$
R_{z}(0)-R_{z}(t)=t I_{z}^{(1)}+t^{2} I_{z}^{(2)}+\Psi_{z}^{0}(t)
$$

where the term $I_{z}^{(2)}=-\left(\Omega_{z}(0) T_{\delta}^{(1)}\right)^{2} R_{z}(0)+\Omega_{z}(0) T_{\delta}^{(2)} R_{z}(0)$ does not depend on $t$, and $\Psi_{z}^{0}(t)$ is of the form

$$
\begin{aligned}
\Psi_{z}^{0}(t)=t^{2}\left(\Omega_{z}(0) T_{\delta}^{(1)}\right)^{2} \Omega_{z}(0) T_{\delta}(t) R_{z}(t) & -t^{3} \Omega_{z}(0) T_{\delta}^{(1)} \Omega_{z}(0) T_{\delta}^{(2)} R_{z}(t) \\
& -t^{2} \Omega_{z}(0) T_{\delta}^{(2)} \Omega_{z}(0) T_{\delta}(t) R_{z}(t) .
\end{aligned}
$$

We estimate the term (3.12) with the help of (3.10) and (3.3):

$$
\begin{aligned}
\left\|\Psi_{z}^{0}(t)\right\|_{\mathfrak{H} \rightarrow \mathfrak{H}} \leq 25 \sqrt{2}(2 \delta)^{-1} & \left(t^{2} 11^{2}\left\|T_{\delta}^{(1)}\right\|_{\mathfrak{d} \rightarrow \mathfrak{d}}^{2}\left\|T_{\delta}(t)\right\|_{\mathfrak{d} \rightarrow \mathfrak{d}}\right. \\
& \left.+|t|^{3} 11\left\|T_{\delta}^{(1)}\right\|_{\mathfrak{d} \rightarrow \mathfrak{d}}\left\|T_{\delta}^{(2)}\right\|_{\mathfrak{d} \rightarrow \mathfrak{d}}+t^{2} 11\left\|T_{\delta}^{(2)}\right\|_{\mathfrak{d} \rightarrow \mathfrak{d}}\left\|T_{\delta}(t)\right\|_{\mathfrak{d} \rightarrow \mathfrak{d}}\right) .
\end{aligned}
$$

Combining this with (3.5)-(3.7), we arrive at

$$
\left\|\Psi_{z}^{0}(t)\right\|_{\mathfrak{H} \rightarrow \mathfrak{H}} \leq \beta_{0}|t|^{3} \delta^{-5 / 2}\left\|X_{1}\right\|^{3}, \quad z \in \Gamma_{\delta}, \quad|t| \leq t^{0}
$$

Recall that by $\beta$ (with indices) we denote various absolute constants. 
3.4. The difference of resolvents. More accurate representation. We substitute (3.12) in (3.11) and use (3.8) once again. Then

$$
\begin{aligned}
R_{z}(0)-R_{z}(t) & =t I_{z}^{(1)}+t^{2} I_{z}^{(2)}+t^{3} I_{z}^{(3)}-t^{3}\left(\Omega_{z}(0) T_{\delta}^{(1)}\right)^{3} \Omega_{z}(0) T_{\delta}(t) R_{z}(t) \\
& +t^{4}\left(\Omega_{z}(0) T_{\delta}^{(1)}\right)^{2} \Omega_{z}(0) T_{\delta}^{(2)} R_{z}(t)+t^{3} \Omega_{z}(0) T_{\delta}^{(1)} \Omega_{z}(0) T_{\delta}^{(2)} \Omega_{z}(0) T_{\delta}(t) R_{z}(t) \\
& +t^{3} \Omega_{z}(0) T_{\delta}^{(2)} \Omega_{z}(0) T_{\delta}^{(1)} \Omega_{z}(0) T_{\delta}(t) R_{z}(t)-t^{4}\left(\Omega_{z}(0) T_{\delta}^{(2)}\right)^{2} R_{z}(t),
\end{aligned}
$$

where the term

$$
I_{z}^{(3)}=\left(\Omega_{z}(0) T_{\delta}^{(1)}\right)^{3} R_{z}(0)-\Omega_{z}(0) T_{\delta}^{(1)} \Omega_{z}(0) T_{\delta}^{(2)} R_{z}(0)-\Omega_{z}(0) T_{\delta}^{(2)} \Omega_{z}(0) T_{\delta}^{(1)} R_{z}(0)
$$

does not depend on $t$. Iterating for the last time, we arrive at the relation

$$
R_{z}(0)-R_{z}(t)=t I_{z}^{(1)}+t^{2} I_{z}^{(2)}+t^{3} I_{z}^{(3)}+t^{4} I_{z}^{(4)}+\widetilde{\Psi}_{z}(t)
$$

where the term

$$
\begin{aligned}
I_{z}^{(4)}= & -\left(\Omega_{z}(0) T_{\delta}^{(1)}\right)^{4} R_{z}(0)+\left(\Omega_{z}(0) T_{\delta}^{(1)}\right)^{2} \Omega_{z}(0) T_{\delta}^{(2)} R_{z}(0) \\
& +\Omega_{z}(0) T_{\delta}^{(1)} \Omega_{z}(0) T_{\delta}^{(2)} \Omega_{z}(0) T_{\delta}^{(1)} R_{z}(0) \\
& +\Omega_{z}(0) T_{\delta}^{(2)}\left(\Omega_{z}(0) T_{\delta}^{(1)}\right)^{2} R_{z}(0)-\left(\Omega_{z}(0) T_{\delta}^{(2)}\right)^{2} R_{z}(0)
\end{aligned}
$$

does not depend on $t$, and $\widetilde{\Psi}_{z}(t)$ is given by

$$
\begin{aligned}
\widetilde{\Psi}_{z}(t)= & t^{4}\left(\Omega_{z}(0) T_{\delta}^{(1)}\right)^{4} \Omega_{z}(0) T_{\delta}(t) R_{z}(t)-t^{5}\left(\Omega_{z}(0) T_{\delta}^{(1)}\right)^{3} \Omega_{z}(0) T_{\delta}^{(2)} R_{z}(t) \\
& -t^{4}\left(\Omega_{z}(0) T_{\delta}^{(1)}\right)^{2} \Omega_{z}(0) T_{\delta}^{(2)} \Omega_{z}(0) T_{\delta}(t) R_{z}(t) \\
& -t^{4} \Omega_{z}(0) T_{\delta}^{(1)} \Omega_{z}(0) T_{\delta}^{(2)} \Omega_{z}(0) T_{\delta}^{(1)} \Omega_{z}(0) T_{\delta}(t) R_{z}(t) \\
& +t^{5} \Omega_{z}(0) T_{\delta}^{(1)}\left(\Omega_{z}(0) T_{\delta}^{(2)}\right)^{2} R_{z}(t)-t^{4} \Omega_{z}(0) T_{\delta}^{(2)}\left(\Omega_{z}(0) T_{\delta}^{(1)}\right)^{2} \Omega_{z}(0) T_{\delta}(t) R_{z}(t) \\
& +t^{5} \Omega_{z}(0) T_{\delta}^{(2)} \Omega_{z}(0) T_{\delta}^{(1)} \Omega_{z}(0) T_{\delta}^{(2)} R_{z}(t)+t^{4}\left(\Omega_{z}(0) T_{\delta}^{(2)}\right)^{2} \Omega_{z}(0) T_{\delta}(t) R_{z}(t)
\end{aligned}
$$

We estimate the term $\widetilde{\Psi}_{z}(t)$ with the help of (3.10) and (3.3):

$$
\begin{aligned}
&\left\|\widetilde{\Psi}_{z}(t)\right\|_{\mathfrak{H} \rightarrow \mathfrak{H}} \leq 25 \sqrt{2}(2 \delta)^{-1}\left(t^{4} 11^{4}\left\|T_{\delta}^{(1)}\right\|_{\mathfrak{d} \rightarrow \mathfrak{d}}^{4}\left\|T_{\delta}(t)\right\|_{\mathfrak{d} \rightarrow \mathfrak{d}}\right. \\
&+|t|^{5} 11^{3}\left\|T_{\delta}^{(1)}\right\|_{\mathfrak{d} \rightarrow \mathfrak{d}}^{3}\left\|T_{\delta}^{(2)}\right\|_{\mathfrak{d} \rightarrow \mathfrak{d}}+3 t^{4} 11^{3}\left\|T_{\delta}^{(1)}\right\|_{\mathfrak{d} \rightarrow \mathfrak{d}}^{2}\left\|T_{\delta}^{(2)}\right\|_{\mathfrak{d} \rightarrow \mathfrak{d}}\left\|T_{\delta}(t)\right\|_{\mathfrak{d} \rightarrow \mathfrak{d}} \\
&\left.\quad+2|t|^{5} 11^{2}\left\|T_{\delta}^{(1)}\right\|_{\mathfrak{d} \rightarrow \mathfrak{d}}\left\|T_{\delta}^{(2)}\right\|_{\mathfrak{d} \rightarrow \mathfrak{d}}^{2}+t^{4} 11^{2}\left\|T_{\delta}^{(2)}\right\|_{\mathfrak{d} \rightarrow \mathfrak{d}}^{2}\left\|T_{\delta}(t)\right\|_{\mathfrak{d} \rightarrow \mathfrak{d}}\right) .
\end{aligned}
$$

Combining this with (3.5)-(3.7), we obtain

$$
\left\|\widetilde{\Psi}_{z}(t)\right\|_{\mathfrak{H} \rightarrow \mathfrak{H}} \leq \widetilde{\beta}|t|^{5} \delta^{-7 / 2}\left\|X_{1}\right\|^{5}, \quad z \in \Gamma_{\delta}, \quad|t| \leq t^{0} .
$$

$\S 4$. Error estimates in approximations for $F(t)$ And $A(t) F(t)$

4.1. The known results. In [BSu1, Chapter 1, §4], approximations for the operatorvalued functions $F(t)$ and $A(t) F(t)$ were obtained. Namely, the following theorem was proved.

Theorem 4.1. Let $A(t)$ be the operator family satisfying the assumptions of Subsection 1.1. Let $P$ be the orthogonal projection of the space $\mathfrak{H}$ onto the subspace $\mathfrak{N}=\operatorname{Ker} X_{0}$, and let $S$ be the spectral germ of the operator family $A(t)$ at $t=0$. Let $t^{0}$ be the number defined in (1.2). Then

$$
\begin{array}{rlrl}
\|F(t)-P\|_{\mathfrak{H} \rightarrow \mathfrak{H}} & \leq C_{1}|t|, & & |t| \leq t^{0}, \\
\left\|A(t) F(t)-t^{2} S P\right\|_{\mathfrak{H} \rightarrow \mathfrak{H}} & \leq C_{2}|t|^{3}, & |t| \leq t^{0} .
\end{array}
$$


The constants $C_{1}$ and $C_{2}$ are given by

$$
C_{1}=\beta_{1} \delta^{-1 / 2}\left\|X_{1}\right\|, \quad C_{2}=\beta_{2} \delta^{-1 / 2}\left\|X_{1}\right\|^{3},
$$

where $\beta_{1}$ and $\beta_{2}$ are some absolute constants.

In $\mathrm{BSu} 2, \S 2,4]$, more accurate approximations were found. We formulate the corresponding result.

Theorem 4.2. Under the assumptions of Theorem 4.1, let $F_{1}$ be the operator defined by (2.6), and let $K$ be the operator defined in (2.19), (2.22), (2.28). Then

$$
\begin{array}{rlrl}
\left\|F(t)-P-t F_{1}\right\|_{\mathfrak{H} \rightarrow \mathfrak{H}} & \leq C_{3} t^{2}, & & |t| \leq t^{0}, \\
\left\|A(t) F(t)-t^{2} S P-t^{3} K\right\|_{\mathfrak{H} \rightarrow \mathfrak{H}} \leq C_{4} t^{4}, & & |t| \leq t^{0} .
\end{array}
$$

The constants $C_{3}$ and $C_{4}$ are given by

$$
C_{3}=\beta_{3} \delta^{-1}\left\|X_{1}\right\|^{2}, \quad C_{4}=\beta_{4} \delta^{-1}\left\|X_{1}\right\|^{4},
$$

where $\beta_{3}$ and $\beta_{4}$ are some absolute constants.

4.2. Estimate for the operator $F_{3}(t)$. Now we approximate the operator $F(t)$ with an error of $O\left(|t|^{3}\right)$. As in [BSu1, BSu2, we start with the representation

$$
F(t)-P=\frac{1}{2 \pi i} \int_{\Gamma_{\delta}}\left(R_{z}(0)-R_{z}(t)\right) d z
$$

and substitute (3.11) in (4.4). Then

$$
F(t)=P+t F_{1}+t^{2} F_{2}+F_{3}(t),
$$

where

$$
\begin{aligned}
F_{1} & =\frac{1}{2 \pi i} \int_{\Gamma_{\delta}} I_{z}^{(1)} d z, \quad F_{2}=\frac{1}{2 \pi i} \int_{\Gamma_{\delta}} I_{z}^{(2)} d z, \\
F_{3}(t) & =\frac{1}{2 \pi i} \int_{\Gamma_{\delta}} \Psi_{z}^{0}(t) d z .
\end{aligned}
$$

From (3.13) and (4.7) it follows that

$$
\left\|F_{3}(t)\right\|_{\mathfrak{H} \rightarrow \mathfrak{H}} \leq \frac{2 \delta+2 \pi \delta}{2 \pi} \beta_{0}|t|^{3} \delta^{-5 / 2}\left\|X_{1}\right\|^{3}, \quad|t| \leq t^{0} .
$$

Comparing (4.5)-(4.8) and (2.1), (2.6), (2.13), we see that the operators $F_{1}$ and $F_{2}$ defined by (4.6) coincide with the operators (2.6) and (2.13), respectively. We have proved the following theorem.

Theorem 4.3. The spectral projection $F(t)=F(t, \delta)$ admits the representation (4.5), where $F_{1}$ and $F_{2}$ are defined by (2.6) and (2.13), respectively, and the error term $F_{3}(t)$ satisfies the estimate

$$
\left\|F_{3}(t)\right\|_{\mathfrak{H} \rightarrow \mathfrak{H}} \leq C_{5}|t|^{3}, \quad|t| \leq t^{0}
$$

where

$$
C_{5}=\left(1+\pi^{-1}\right) \beta_{0} \delta^{-3 / 2}\left\|X_{1}\right\|^{3}
$$


4.3. Estimate for the operator $\Psi(t)$. Now, we approximate the operator $A(t) F(t)$ with an error term of order $O\left(|t|^{5}\right)$. As in $\mathrm{BSu} 1, \mathrm{BSu} 2$, we start with the representation

$$
A(t) F(t)=\frac{1}{2 \pi i} \int_{\Gamma_{\delta}} z\left(R_{z}(0)-R_{z}(t)\right) d z .
$$

Substituting (3.14) in (4.11), we obtain

$$
A(t) F(t)=t \mathcal{I}^{(1)}+t^{2} \mathcal{I}^{(2)}+t^{3} \mathcal{I}^{(3)}+t^{4} \mathcal{I}^{(4)}+\Psi(t),
$$

where

$$
\begin{aligned}
\mathcal{I}^{(j)} & =\frac{1}{2 \pi i} \int_{\Gamma_{\delta}} z I_{z}^{(j)} d z, \quad j=1,2,3,4, \\
\Psi(t) & =\frac{1}{2 \pi i} \int_{\Gamma_{\delta}} z \widetilde{\Psi}_{z}(t) d z .
\end{aligned}
$$

From (3.15), (4.13), and the estimate $|z| \leq 2 \delta$ it follows that

$$
\|\Psi(t)\|_{\mathfrak{H} \rightarrow \mathfrak{H}} \leq \frac{(2 \delta+2 \pi \delta) 2 \delta}{2 \pi} \widetilde{\beta}|t|^{5} \delta^{-7 / 2}\left\|X_{1}\right\|^{5}, \quad|t| \leq t^{0}
$$

Comparing (4.12), (4.14), and (2.16), we see that $\mathcal{I}^{(1)}=\mathbf{0}, \mathcal{I}^{(2)}=S P, \mathcal{I}^{(3)}=K$, $\mathcal{I}^{(4)}=W$. We arrive at the following result.

Theorem 4.4. The operator $A(t) F(t)$ admits the representation

$$
A(t) F(t)=t^{2} S P+t^{3} K+t^{4} W+\Psi(t),
$$

where $S$ is the spectral germ defined in Subsection 1.3, the operator $K$ is defined in (2.19), (2.22), (2.28), and $W$ is defined in accordance with (2.31), (2.35), (2.37), and (2.48), (2.49). The error term $\Psi(t)$ satisfies the estimate

$$
\|\Psi(t)\|_{\mathfrak{H} \rightarrow \mathfrak{H}} \leq C_{6}|t|^{5}, \quad|t| \leq t^{0},
$$

where

$$
C_{6}=2\left(1+\pi^{-1}\right) \widetilde{\beta} \delta^{-3 / 2}\left\|X_{1}\right\|^{5} .
$$

\section{§5. Approximation of the operator exponential}

\subsection{Preliminaries. Assume that}

$$
A(t) \geq c_{*} t^{2} I, \quad c_{*}>0, \quad|t| \leq t^{0} .
$$

This is equivalent to the estimates $\lambda_{l}(t) \geq c_{*} t^{2}, l=1, \ldots, n,|t| \leq t^{0}$, for the eigenvalues of $A(t)$. Combining this with (1.21), we see that $\gamma_{l} \geq c_{*}, l=1, \ldots, n$, whence, by (1.30), the spectral germ $S$ satisfies the estimate

$$
S \geq c_{*} I_{\mathfrak{N}}
$$

Our goal is to approximate the operator exponential $e^{-A(t) \tau}$ in the operator norm in $\mathfrak{H}$ as $\tau \rightarrow+\infty$ under the additional condition (5.1). In [Su1, Su2, the principal term of such an approximation was obtained and an error estimate of order $O\left(\tau^{-1 / 2}\right)$ was proved; in [V], a more accurate approximation with an error term of order $O\left(\tau^{-1}\right)$ was deduced. Now we establish an even more accurate approximation with an error term of $O\left(\tau^{-3 / 2}\right)$. As in [Su1, $\underline{\mathrm{Su} 2}, \mathrm{~V}$, the method of proof is based on using of approximations in the operator norm for the spectral projection $F(t)$ and the operator $A(t) F(t)$ (see Theorems 4.1, 4.2, 4.3, 4.4). 
We formulate the necessary statements from [Su2] and [V]. First, from (5.1) and (5.2) it follows that

$$
\begin{aligned}
\left\|e^{-A(t) \tau}\right\|_{\mathfrak{H} \rightarrow \mathfrak{H}} \leq e^{-c_{*} t^{2} \tau}, \quad \tau \geq 0, \quad|t| \leq t^{0}, \\
\left\|e^{-t^{2} S \tau} P\right\|_{\mathfrak{H} \rightarrow \mathfrak{H}} \leq e^{-c_{*} t^{2} \tau}, \quad \tau \geq 0, \quad t \in \mathbb{R} .
\end{aligned}
$$

We represent the operator $e^{-A(t) \tau}$ as

$$
e^{-A(t) \tau}=e^{-A(t) \tau} F(t)+e^{-A(t) \tau} F(t)^{\perp} .
$$

By (1.2), for $|t| \leq t^{0}$ the operator $F(t)^{\perp}$ is the spectral projection of $A(t)$ for the semiaxis $[3 \delta, \infty)$, whence

$$
\left\|e^{-A(t) \tau} F(t)^{\perp}\right\|_{\mathfrak{H} \rightarrow \mathfrak{H}} \leq e^{-3 \delta \tau} \leq(3 \delta \tau)^{-3 / 2}, \quad \tau>0, \quad|t| \leq t^{0} .
$$

This estimate shows that the problem of approximation of the operator $e^{-A(t) \tau}$ is of "threshold" nature: the main contribution is given by the operator $e^{-A(t) \tau} F(t)$, which corresponds to the part of the spectrum of $A(t)$ near its bottom.

Next, we denote

$$
e^{-A(t) \tau} F(t)-e^{-t^{2} S \tau} P=: \Pi(t, \tau) .
$$

In [Su2, (2.11)], on the basis of (4.1) and (4.2) it was shown that

$$
\|\Pi(t, \tau)\|_{\mathfrak{H} \rightarrow \mathfrak{H}} \leq\left(2 C_{1}|t|+C_{2}|t|^{3} \tau\right) e^{-c_{*} t^{2} \tau}, \quad \tau \geq 0, \quad|t| \leq t^{0}
$$

where the constants $C_{1}, C_{2}$ are given in (4.3). From (5.8) it follows that $\|\Pi(t, \tau)\|$ satisfies an estimate of order $O\left(\tau^{-1 / 2}\right)$ for $|t| \leq t^{0}$ :

$$
\|\Pi(t, \tau)\|_{\mathfrak{H} \rightarrow \mathfrak{H}} \leq \tau^{-1 / 2}\left(2 C_{1} c_{*}^{-1 / 2} c(1 / 2)+C_{2} c_{*}^{-3 / 2} c(3 / 2)\right), \quad \tau>0, \quad|t| \leq t^{0} .
$$

Here

$$
c(s):=\max _{x \geq 0} x^{s} e^{-x}, \quad s>0 .
$$

In $[\mathrm{V}, \S 2]$, a more accurate approximation for the operator (5.7) was obtained:

$$
\Pi(t, \tau)=\mathcal{K}^{(1)}(t, \tau)+J(t, \tau),
$$

where the corrector $\mathcal{K}^{(1)}(t, \tau)$ is given by

$$
\begin{aligned}
\mathcal{K}^{(1)}(t, \tau) & =t\left(Z e^{-t^{2} S \tau} P+e^{-t^{2} S \tau} P Z^{*}\right)-t^{3} \mathcal{M}(t, \tau), \\
\mathcal{M}(t, \tau) & =\int_{0}^{\tau} e^{-t^{2} S(\tau-\rho)} N e^{-t^{2} S \rho} P d \rho .
\end{aligned}
$$

Here $Z$ is the operator defined in Subsection 1.2, and $N$ is the operator (2.28). Note that the operators $\mathcal{M}(t, \tau)$ and $\mathcal{K}^{(1)}(t, \tau)$ are selfadjoint in $\mathfrak{H}$. By $(5.4),(1.7)$, and $(2.29)$, the operator (5.10) satisfies the estimate

$$
\begin{aligned}
\left\|\mathcal{K}^{(1)}(t, \tau)\right\|_{\mathfrak{H} \rightarrow \mathfrak{H}} & \leq\left(2\|Z\||t|+\|N\||t|^{3} \tau\right) e^{-c_{*} t^{2} \tau} \\
& \leq(2 \delta)^{-1 / 2}\left(\left\|X_{1}\right\||t|+\left\|X_{1}\right\|^{3}|t|^{3} \tau\right) e^{-c_{*} t^{2} \tau}, \quad \tau \geq 0, \quad t \in \mathbb{R} .
\end{aligned}
$$

This shows that $\left\|\mathcal{K}^{(1)}(t, \tau)\right\|=O\left(\tau^{-1 / 2}\right)$.

As was proved in $[\mathrm{V}$, the error term $J(t, \tau)$ is subject to the estimate

$$
\|J(t, \tau)\|_{\mathfrak{H} \rightarrow \mathfrak{H}} \leq\left(\mathcal{C}_{1} t^{2}+\mathcal{C}_{2} t^{4} \tau+\mathcal{C}_{3} t^{6} \tau^{2}\right) e^{-c_{*} t^{2} \tau}, \quad \tau \geq 0, \quad|t| \leq t^{0},
$$

where

$$
\mathcal{C}_{1}=\widehat{\beta}_{1} \delta^{-1}\left\|X_{1}\right\|^{2}, \quad \mathcal{C}_{2}=\widehat{\beta}_{2} \delta^{-1}\left\|X_{1}\right\|^{4}, \quad \mathcal{C}_{3}=\widehat{\beta}_{3} \delta^{-1}\left\|X_{1}\right\|^{6} .
$$

Then $\|J(t, \tau)\|$ is of order $O\left(\tau^{-1}\right)$ for $|t| \leq t^{0}$ :

$$
\|J(t, \tau)\|_{\mathfrak{H} \rightarrow \mathfrak{H}} \leq \tau^{-1}\left(\mathcal{C}_{1} c_{*}^{-1} c(1)+\mathcal{C}_{2} c_{*}^{-2} c(2)+\mathcal{C}_{3} c_{*}^{-3} c(3)\right), \quad \tau>0, \quad|t| \leq t^{0} .
$$


5.2. Analysis of the operator $P^{\perp} e^{-A(t) \tau} F(t)$. The operator (5.7) is represented as

$$
\begin{aligned}
& \Pi(t, \tau)=P^{\perp} e^{-A(t) \tau} F(t)+\Sigma(t, \tau), \\
& \Sigma(t, \tau)=P e^{-A(t) \tau} F(t)-e^{-t^{2} S \tau} P .
\end{aligned}
$$

We start with the analysis of the operator $P^{\perp} e^{-A(t) \tau} F(t)$. By (4.5), we have

$$
P^{\perp} F(t)=(F(t)-P) F(t)=\left(t F_{1}+t^{2} F_{2}+F_{3}(t)\right) F(t),
$$

whence

$$
P^{\perp} e^{-A(t) \tau} F(t)=t F_{1} e^{-A(t) \tau} F(t)+t^{2} F_{2} e^{-A(t) \tau} F(t)+F_{3}(t) e^{-A(t) \tau} F(t) .
$$

The last term on the right-hand side of (5.17) is estimated with the help of (4.9) and (5.3):

$$
\left\|F_{3}(t) e^{-A(t) \tau} F(t)\right\|_{\mathfrak{H} \rightarrow \mathfrak{H}} \leq C_{5}|t|^{3} e^{-c_{*} t^{2} \tau} \leq \mathcal{C}_{4} \tau^{-3 / 2}, \quad \tau>0, \quad|t| \leq t^{0},
$$

where

$$
\mathcal{C}_{4}=C_{5} c_{*}^{-3 / 2} c(3 / 2)=\left(1+\pi^{-1}\right) \beta_{0} c(3 / 2) \delta^{-3 / 2}\left\|X_{1}\right\|^{3} c_{*}^{-3 / 2} .
$$

By (5.7), we rewrite the second term on the right-hand side of (5.17) as

$$
t^{2} F_{2} e^{-A(t) \tau} F(t)=t^{2} F_{2}\left(e^{-t^{2} S \tau} P+\Pi(t, \tau)\right) .
$$

By (5.8) and (2.14), we have

$$
\left\|t^{2} F_{2} \Pi(t, \tau)\right\|_{\mathfrak{H} \rightarrow \mathfrak{H}} \leq\left\|F_{2}\right\|\left(2 C_{1}|t|^{3}+C_{2}|t|^{5} \tau\right) e^{-c_{*} t^{2} \tau} \leq \mathcal{C}_{5} \tau^{-3 / 2}, \quad \tau>0, \quad|t| \leq t^{0},
$$

where

$$
\begin{aligned}
\mathcal{C}_{5} & =3(4 \delta)^{-1}\left\|X_{1}\right\|^{2}\left(2 C_{1} c_{*}^{-3 / 2} c(3 / 2)+C_{2} c_{*}^{-5 / 2} c(5 / 2)\right) \\
& =3 / 4 \delta^{-3 / 2}\left(2 \beta_{1} c(3 / 2)\left\|X_{1}\right\|^{3} c_{*}^{-3 / 2}+\beta_{2} c(5 / 2)\left\|X_{1}\right\|^{5} c_{*}^{-5 / 2}\right) .
\end{aligned}
$$

Here we have used relations (4.3).

By (5.7), (5.9), and (5.10), the first term on the right-hand side of (5.17) can be transformed as follows:

$$
\begin{aligned}
t F_{1} e^{-A(t) \tau} F(t)= & t F_{1} e^{-t^{2} S \tau} P+t^{2} F_{1}\left(Z e^{-t^{2} S \tau} P+e^{-t^{2} S \tau} P Z^{*}\right) \\
& -t^{4} F_{1} \mathcal{M}(t, \tau)+t F_{1} J(t, \tau) .
\end{aligned}
$$

The last term on the right-hand side of (5.23) is estimated with the help of (5.13) and (2.7):

$$
\begin{array}{r}
\left\|t F_{1} J(t, \tau)\right\|_{\mathfrak{H} \rightarrow \mathfrak{H}} \leq\left\|F_{1}\right\|\left(\mathcal{C}_{1}|t|^{3}+\mathcal{C}_{2}|t|^{5} \tau+\mathcal{C}_{3}|t|^{7} \tau^{2}\right) e^{-c_{*} t^{2} \tau} \leq \mathcal{C}_{6} \tau^{-3 / 2} \\
\tau>0, \quad|t| \leq t^{0}
\end{array}
$$

where

$$
\begin{aligned}
\mathcal{C}_{6} & =(2 \delta)^{-1 / 2}\left\|X_{1}\right\|\left(\mathcal{C}_{1} c_{*}^{-3 / 2} c(3 / 2)+\mathcal{C}_{2} c_{*}^{-5 / 2} c(5 / 2)+\mathcal{C}_{3} c_{*}^{-7 / 2} c(7 / 2)\right) \\
& =2^{-1 / 2} \delta^{-3 / 2}\left(\widehat{\beta}_{1} c(3 / 2)\left\|X_{1}\right\|^{3} c_{*}^{-3 / 2}+\widehat{\beta}_{2} c(5 / 2)\left\|X_{1}\right\|^{5} c_{*}^{-5 / 2}+\widehat{\beta}_{3} c(7 / 2)\left\|X_{1}\right\|^{7} c_{*}^{-7 / 2}\right) .
\end{aligned}
$$

We have used relations (5.14).

As a result, (5.17), (5.20), and (5.23) imply

$$
\begin{aligned}
P^{\perp} e^{-A(t) \tau} F(t)=t F_{1} e^{-t^{2} S \tau} P & +t^{2} F_{1}\left(Z e^{-t^{2} S \tau} P+e^{-t^{2} S \tau} P Z^{*}\right) \\
& -t^{4} F_{1} \mathcal{M}(t, \tau)+t^{2} F_{2} e^{-t^{2} S \tau} P+J_{1}(t, \tau),
\end{aligned}
$$


where $J_{1}(t, \tau)=t F_{1} J(t, \tau)+t^{2} F_{2} \Pi(t, \tau)+F_{3}(t) e^{-A(t) \tau} F(t)$. From (5.18), (5.21), and (5.24) it follows that

$$
\left\|J_{1}(t, \tau)\right\| \leq\left(\mathcal{C}_{4}+\mathcal{C}_{5}+\mathcal{C}_{6}\right) \tau^{-3 / 2}, \quad \tau>0, \quad|t| \leq t^{0} .
$$

Now we transform (5.26) by using (2.6) and (2.13). The properties of the operators $Z$ and $Z_{2}$ (see Subsections 1.2, 1.4) show that

$$
F_{1} P=Z, \quad F_{1} Z=Z^{*} Z, \quad F_{2} P=Z_{2}-Z^{*} Z .
$$

Therefore, the representation (5.26) takes the form

$$
\begin{aligned}
P^{\perp} e^{-A(t) \tau} F(t)=t Z e^{-t^{2} S \tau} P & +t^{2} Z e^{-t^{2} S \tau} P Z^{*} \\
& -t^{4} Z \mathcal{M}(t, \tau)+t^{2} Z_{2} e^{-t^{2} S \tau} P+J_{1}(t, \tau) .
\end{aligned}
$$

5.3. Analysis of the operator $\Sigma(t, \tau)$. By $[\underline{S u 2}, \S 2]$, the operator (5.16) satisfies

(5.30) $\Sigma(t, \tau)=e^{-t^{2} S \tau} P(F(t)-P)-\int_{0}^{\tau} e^{-t^{2} S(\tau-\rho)} P\left(A(t) F(t)-t^{2} S P\right) e^{-A(t) \rho} F(t) d \rho$.

By (4.5), the first term on the right-hand side of (5.30) can be written as

$$
e^{-t^{2} S \tau} P(F(t)-P)=e^{-t^{2} S \tau} P\left(t F_{1}+t^{2} F_{2}\right)+e^{-t^{2} S \tau} P F_{3}(t) .
$$

From (4.9) and (5.4) it follows that

$$
\left\|e^{-t^{2} S \tau} P F_{3}(t)\right\|_{\mathfrak{H} \rightarrow \mathfrak{H}} \leq C_{5}|t|^{3} e^{-c_{*} t^{2} \tau} \leq \mathcal{C}_{4} \tau^{-3 / 2}, \quad \tau>0, \quad|t| \leq t^{0}
$$

cf. (5.18). By (4.15), the second term on the right-hand side of (5.30) can be written as

$$
\begin{aligned}
\int_{0}^{\tau} e^{-t^{2} S(\tau-\rho)} P\left(A(t) F(t)-t^{2} S P\right) e^{-A(t) \rho} F(t) d \rho \\
\quad=\int_{0}^{\tau} e^{-t^{2} S(\tau-\rho)} P\left(t^{3} K+t^{4} W+\Psi(t)\right) e^{-A(t) \rho} F(t) d \rho .
\end{aligned}
$$

From (4.16), (5.3), and (5.4) it follows that

$$
\begin{aligned}
\left\|\int_{0}^{\tau} e^{-t^{2} S(\tau-\rho)} P \Psi(t) e^{-A(t) \rho} F(t) d \rho\right\| \leq C_{6}|t|^{5} \tau e^{-c_{*} t^{2} \tau} & \leq \mathcal{C}_{7} \tau^{-3 / 2}, \\
\tau & >0, \quad|t| \leq t^{0},
\end{aligned}
$$

where

$$
\mathcal{C}_{7}=C_{6} c_{*}^{-5 / 2} c(5 / 2)=2\left(1+\pi^{-1}\right) \widetilde{\beta} \delta^{-3 / 2}\left\|X_{1}\right\|^{5} c_{*}^{-5 / 2} c(5 / 2) .
$$

Next, by (5.7), we can represent the term containing $W$ on the right-hand side of (5.33) as

$$
\int_{0}^{\tau} e^{-t^{2} S(\tau-\rho)} P t^{4} W e^{-A(t) \rho} F(t) d \rho=\int_{0}^{\tau} e^{-t^{2} S(\tau-\rho)} P t^{4} W\left(e^{-t^{2} S \rho} P+\Pi(t, \rho)\right) d \rho .
$$

By (5.4) and (5.8),

$$
\begin{aligned}
& \left\|\int_{0}^{\tau} e^{-t^{2} S(\tau-\rho)} P t^{4} W \Pi(t, \rho) d \rho\right\| \\
& \quad \leq\|W\|\left(2 C_{1}|t|^{5} \tau+C_{2}|t|^{7} \frac{\tau^{2}}{2}\right) e^{-c_{*} t^{2} \tau}, \quad \tau \geq 0, \quad|t| \leq t^{0} .
\end{aligned}
$$

Combining this with (2.51) and (4.3), we obtain

$$
\left\|\int_{0}^{\tau} e^{-t^{2} S(\tau-\rho)} P t^{4} W \Pi(t, \rho) d \rho\right\| \leq \mathcal{C}_{8} \tau^{-3 / 2}, \quad \tau>0, \quad|t| \leq t^{0},
$$


where

$$
\begin{aligned}
\mathcal{C}_{8} & =5(2 \delta)^{-1}\left\|X_{1}\right\|^{4}\left(2 C_{1} c_{*}^{-5 / 2} c(5 / 2)+\frac{1}{2} C_{2} c_{*}^{-7 / 2} c(7 / 2)\right) \\
& =5 / 2 \delta^{-3 / 2}\left(2 \beta_{1} c(5 / 2)\left\|X_{1}\right\|^{5} c_{*}^{-5 / 2}+\frac{1}{2} \beta_{2} c(7 / 2)\left\|X_{1}\right\|^{7} c_{*}^{-7 / 2}\right) .
\end{aligned}
$$

Now, we transform the term containing $K$ on the right-hand side of (5.33) with the help of (5.7), (5.9), and (5.10):

$$
\begin{aligned}
\int_{0}^{\tau} e^{-t^{2} S(\tau-\rho)} P t^{3} K e^{-A(t) \rho} F(t) d \rho \\
=\int_{0}^{\tau} e^{-t^{2} S(\tau-\rho)} P t^{3} K\left(e^{-t^{2} S \rho} P+t Z e^{-t^{2} S \rho} P+t e^{-t^{2} S \rho} P Z^{*}-t^{3} \mathcal{M}(t, \rho)\right) d \rho \\
\quad+\int_{0}^{\tau} e^{-t^{2} S(\tau-\rho)} P t^{3} K J(t, \rho) d \rho
\end{aligned}
$$

The following estimate of the last term on the right-hand side of (5.39) is a consequence of (5.4) and (5.13):

$$
\left\|\int_{0}^{\tau} e^{-t^{2} S(\tau-\rho)} P t^{3} K J(t, \rho) d \rho\right\| \leq\|K\|\left(\mathcal{C}_{1}|t|^{5} \tau+\mathcal{C}_{2}|t|^{7} \frac{\tau^{2}}{2}+\mathcal{C}_{3}|t|^{9} \frac{\tau^{3}}{3}\right) e^{-c_{*} t^{2} \tau}
$$

for $\tau \geq 0$ and $|t| \leq t^{0}$. Combining this with (2.30) and (5.14), we get

$$
\left\|\int_{0}^{\tau} e^{-t^{2} S(\tau-\rho)} P t^{3} K J(t, \rho) d \rho\right\| \leq \mathcal{C}_{9} \tau^{-3 / 2}, \quad \tau>0, \quad|t| \leq t^{0},
$$

where

$$
\begin{aligned}
\mathcal{C}_{9}=2(2 \delta)^{-1 / 2}\left\|X_{1}\right\|^{3}\left(\mathcal{C}_{1} c_{*}^{-5 / 2} c(5 / 2)\right. & \left.+\frac{1}{2} \mathcal{C}_{2} c_{*}^{-7 / 2} c(7 / 2)+\frac{1}{3} \mathcal{C}_{3} c_{*}^{-9 / 2} c(9 / 2)\right) \\
=\sqrt{2} \delta^{-3 / 2}\left(\widehat{\beta}_{1} c(5 / 2)\left\|X_{1}\right\|^{5} c_{*}^{-5 / 2}\right. & +\frac{1}{2} \widehat{\beta}_{2} c(7 / 2)\left\|X_{1}\right\|^{7} c_{*}^{-7 / 2} \\
& \left.+\frac{1}{3} \widehat{\beta}_{3} c(9 / 2)\left\|X_{1}\right\|^{9} c_{*}^{-9 / 2}\right) .
\end{aligned}
$$

As a result, relations (5.30), (5.31), (5.33), (5.36), and (5.39) imply

$$
\begin{aligned}
\Sigma(t, \tau)= & e^{-t^{2} S \tau} P\left(t F_{1}+t^{2} F_{2}\right)-\int_{0}^{\tau} e^{-t^{2} S(\tau-\rho)} P t^{3} K e^{-t^{2} S \rho} P d \rho \\
& -\int_{0}^{\tau} e^{-t^{2} S(\tau-\rho)} P t^{3} K\left(t Z e^{-t^{2} S \rho} P+t e^{-t^{2} S \rho} P Z^{*}-t^{3} \mathcal{M}(t, \rho)\right) d \rho \\
& -\int_{0}^{\tau} e^{-t^{2} S(\tau-\rho)} P t^{4} W e^{-t^{2} S \rho} P d \rho+J_{2}(t, \tau),
\end{aligned}
$$

where

$$
\begin{aligned}
J_{2}(t, \tau)= & e^{-t^{2} S \tau} P F_{3}(t)-\int_{0}^{\tau} e^{-t^{2} S(\tau-\rho)} P \Psi(t) e^{-A(t) \rho} F(t) d \rho \\
& -\int_{0}^{\tau} e^{-t^{2} S(\tau-\rho)} P t^{3} K J(t, \rho) d \rho-\int_{0}^{\tau} e^{-t^{2} S(\tau-\rho)} P t^{4} W \Pi(t, \rho) d \rho .
\end{aligned}
$$

From (5.32), (5.34), (5.37), and (5.40) it follows that

$$
\left\|J_{2}(t, \tau)\right\| \leq\left(\mathcal{C}_{4}+\mathcal{C}_{7}+\mathcal{C}_{8}+\mathcal{C}_{9}\right) \tau^{-3 / 2}, \quad \tau>0, \quad|t| \leq t^{0} .
$$


Now we turn to the representation (5.42). From (5.28) we see that $P F_{1}=Z^{*}, P F_{2}=$ $Z_{2}^{*}-Z^{*} Z$. Hence, for the first term on the right-hand side of (5.42) we have

$$
e^{-t^{2} S \tau} P\left(t F_{1}+t^{2} F_{2}\right)=t e^{-t^{2} S \tau} P Z^{*}+t^{2} e^{-t^{2} S \tau} P\left(Z_{2}^{*}-Z^{*} Z\right) .
$$

Combining (2.19) and the properties of the operators $K_{0}$ and $N$ (see Subsection 2.2), we see that

$$
P K P=P N P=N .
$$

By (5.11), the second term on the right-hand side of (5.42) can be written as $-t^{3} \mathcal{M}(t, \tau)$. Next, from (2.19), (2.22), and the properties of the operators $Z, K_{0}$, and $N$ it follows that $P K Z=P K_{0} Z=S P Z^{*} Z$. Therefore, the third term on the right-hand side of (5.42) takes the form

$$
-t^{4} \int_{0}^{\tau} e^{-t^{2} S(\tau-\rho)} S P Z^{*} Z e^{-t^{2} S \rho} P d \rho-t^{4} \mathcal{M}(t, \tau) Z^{*}+t^{6} \int_{0}^{\tau} e^{-t^{2} S(\tau-\rho)} N \mathcal{M}(t, \rho) d \rho .
$$

Finally, combining (2.31) and the properties of the operators $W_{0}, K_{1}$, and $N_{1}$ (see Subsection 2.3), and (2.48), we obtain

$$
P W P=P N_{1} P=N_{1}=N_{1}^{0}-Z^{*} Z S P-S P Z^{*} Z .
$$

This allows us to rewrite the fourth term on the right-hand side of (5.42) as

$$
-t^{4} \int_{0}^{\tau} e^{-t^{2} S(\tau-\rho)} N_{1}^{0} e^{-t^{2} S \rho} P d \rho+t^{4} \int_{0}^{\tau} e^{-t^{2} S(\tau-\rho)} P\left(Z^{*} Z S P+S P Z^{*} Z\right) e^{-t^{2} S \rho} P d \rho .
$$

Taking into account all the transformations described above, we see that the representation (5.42) turns into

$$
\begin{aligned}
\Sigma(t, \tau)= & t e^{-t^{2} S \tau} P Z^{*}+t^{2} e^{-t^{2} S \tau} P\left(Z_{2}^{*}-Z^{*} Z\right)-t^{3} \mathcal{M}(t, \tau)-t^{4} \mathcal{M}(t, \tau) Z^{*} \\
& +t^{6} \int_{0}^{\tau} e^{-t^{2} S(\tau-\rho)} N \mathcal{M}(t, \rho) d \rho-t^{4} \int_{0}^{\tau} e^{-t^{2} S(\tau-\rho)} N_{1}^{0} e^{-t^{2} S \rho} P d \rho \\
& +t^{4} \int_{0}^{\tau} e^{-t^{2} S(\tau-\rho)} Z^{*} Z S P e^{-t^{2} S \rho} P d \rho+J_{2}(t, \tau) .
\end{aligned}
$$

5.4. The final result. Now, from (5.7), (5.15), (5.29), and (5.46) it follows that

$$
e^{-A(t) \tau} F(t)=e^{-t^{2} S \tau} P+\mathcal{K}^{(1)}(t, \tau)+\mathcal{K}^{(2)}(t, \tau)+J_{1}(t, \tau)+J_{2}(t, \tau),
$$

where the "first" corrector $\mathcal{K}^{(1)}(t, \tau)$ is defined by $(5.10)$, and the "second" corrector $\mathcal{K}^{(2)}(t, \tau)$ is the sum of five terms:

$$
\mathcal{K}^{(2)}(t, \tau)=\sum_{j=1}^{5} \mathcal{K}_{j}^{(2)}(t, \tau)
$$

where

$$
\begin{aligned}
& \mathcal{K}_{1}^{(2)}(t, \tau)=t^{2}\left(Z_{2} e^{-t^{2} S \tau} P+e^{-t^{2} S \tau} P Z_{2}^{*}+Z e^{-t^{2} S \tau} P Z^{*}\right), \\
& \mathcal{K}_{2}^{(2)}(t, \tau)=-t^{4}\left(Z \mathcal{M}(t, \tau)+\mathcal{M}(t, \tau) Z^{*}\right), \\
& \mathcal{K}_{3}^{(2)}(t, \tau)=-t^{4} \int_{0}^{\tau} e^{-t^{2} S(\tau-\rho)} N_{1}^{0} e^{-t^{2} S \rho} P d \rho, \\
& \mathcal{K}_{4}^{(2)}(t, \tau)=-t^{2} e^{-t^{2} S \tau} P Z^{*} Z+t^{4} \int_{0}^{\tau} e^{-t^{2} S(\tau-\rho)} Z^{*} Z S P e^{-t^{2} S \rho} P d \rho, \\
& \mathcal{K}_{5}^{(2)}(t, \tau)=t^{6} \int_{0}^{\tau} e^{-t^{2} S(\tau-\rho)} N \mathcal{M}(t, \rho) d \rho .
\end{aligned}
$$


It is easily seen that each of the operators (5.49)-(5.53) is selfadjoint in $\mathfrak{H}$. Also, the operator (5.52) satisfies

$$
\begin{aligned}
\mathcal{K}_{4}^{(2)}(t, \tau) & =-t^{2} Z^{*} Z e^{-t^{2} S \tau} P+t^{4} \int_{0}^{\tau} e^{-t^{2} S(\tau-\rho)} S P Z^{*} Z e^{-t^{2} S \rho} P d \rho \\
& =-t^{2} \frac{\partial}{\partial \tau} \int_{0}^{\tau} e^{-t^{2} S(\tau-\rho)} Z^{*} Z e^{-t^{2} S \rho} P d \rho .
\end{aligned}
$$

We estimate the operators (5.49)-(5.53). From (5.4), (1.7), and (1.16) it follows that

$$
\left\|\mathcal{K}_{1}^{(2)}(t, \tau)\right\|_{\mathfrak{H} \rightarrow \mathfrak{H}} \leq 5(8 \delta)^{-1}\left\|X_{1}\right\|^{2} t^{2} e^{-c_{*} t^{2} \tau}, \quad \tau \geq 0, \quad t \in \mathbb{R} .
$$

Relations (5.4), (5.11), (1.7), and (2.29) imply the inequality

$$
\left\|\mathcal{K}_{2}^{(2)}(t, \tau)\right\|_{\mathfrak{H} \rightarrow \mathfrak{H}} \leq(2 \delta)^{-1}\left\|X_{1}\right\|^{4} t^{4} \tau e^{-c_{*} t^{2} \tau}, \quad \tau \geq 0, \quad t \in \mathbb{R} .
$$

By (5.4) and (2.50), we have

$$
\left\|\mathcal{K}_{3}^{(2)}(t, \tau)\right\|_{\mathfrak{H} \rightarrow \mathfrak{H}} \leq 9(8 \delta)^{-1}\left\|X_{1}\right\|^{4} t^{4} \tau e^{-c_{*} t^{2} \tau}, \quad \tau \geq 0, \quad t \in \mathbb{R}
$$

By (5.4), (1.7), and (1.11), we obtain

$$
\left\|\mathcal{K}_{4}^{(2)}(t, \tau)\right\|_{\mathfrak{H} \rightarrow \mathfrak{H}} \leq(8 \delta)^{-1}\left(\left\|X_{1}\right\|^{2} t^{2}+\left\|X_{1}\right\|^{4} t^{4} \tau\right) e^{-c_{*} t^{2} \tau}, \quad \tau \geq 0, \quad t \in \mathbb{R} .
$$

Finally, the estimate of the operator (5.53) is a consequence of (5.4), (5.11), and (2.29):

$$
\left\|\mathcal{K}_{5}^{(2)}(t, \tau)\right\|_{\mathfrak{H} \rightarrow \mathfrak{H}} \leq(4 \delta)^{-1}\left\|X_{1}\right\|^{6} t^{6} \tau^{2} e^{-c_{*} t^{2} \tau}, \quad \tau \geq 0, \quad t \in \mathbb{R} .
$$

As a result, relations (5.55)-(5.59) imply the following estimate of the operator (5.48):

$$
\begin{array}{r}
\left\|\mathcal{K}^{(2)}(t, \tau)\right\|_{\mathfrak{H} \rightarrow \mathfrak{H}} \leq(4 \delta)^{-1}\left(3\left\|X_{1}\right\|^{2} t^{2}+7\left\|X_{1}\right\|^{4} t^{4} \tau+\left\|X_{1}\right\|^{6} t^{6} \tau^{2}\right) e^{-c_{*} t^{2} \tau}, \\
\tau \geq 0, \quad t \in \mathbb{R} .
\end{array}
$$

This inequality shows that $\left\|\mathcal{K}^{(2)}(t, \tau)\right\|$ is of order $O\left(\tau^{-1}\right)$.

Now the result about approximation of the operator exponential $e^{-A(t) \tau}$ follows from (5.5), (5.47), and estimates (5.6), (5.27), (5.43). We use the notation

$$
\begin{aligned}
J^{0}(t, \tau) & =e^{-A(t) \tau} F(t)^{\perp}+J_{1}(t, \tau)+J_{2}(t, \tau), \\
\mathcal{C}^{0} & =(3 \delta)^{-3 / 2}+2 \mathcal{C}_{4}+\mathcal{C}_{5}+\mathcal{C}_{6}+\mathcal{C}_{7}+\mathcal{C}_{8}+\mathcal{C}_{9} .
\end{aligned}
$$

From (5.19), (5.22), (5.25), (5.35), (5.38), and (5.41), we deduce that the constant $\mathcal{C}^{0}$ is given by

$$
\mathcal{C}^{0}=\delta^{-3 / 2}\left(3^{-3 / 2}+\beta_{1}^{\circ}\left\|X_{1}\right\|^{3} c_{*}^{-3 / 2}+\beta_{2}^{\circ}\left\|X_{1}\right\|^{5} c_{*}^{-5 / 2}+\beta_{3}^{\circ}\left\|X_{1}\right\|^{7} c_{*}^{-7 / 2}+\beta_{4}^{\circ}\left\|X_{1}\right\|^{9} c_{*}^{-9 / 2}\right),
$$

where the $\beta_{j}^{\circ}, j=1,2,3,4$, are some absolute constants. We have proved the following theorem.

Theorem 5.1. We have

$$
e^{-A(t) \tau}=e^{-t^{2} S \tau} P+\mathcal{K}^{(1)}(t, \tau)+\mathcal{K}^{(2)}(t, \tau)+J^{0}(t, \tau)
$$

where the operator $\mathcal{K}^{(1)}(t, \tau)$ is defined by $(5.10)$, and $\mathcal{K}^{(2)}(t, \tau)$ is defined in accordance with (5.48)-(5.53). The operators $\mathcal{K}^{(1)}(t, \tau)$ and $\mathcal{K}^{(2)}(t, \tau)$ satisfy estimates $(5.12)$ and (5.60), respectively. The error term $J^{0}(t, \tau)$ satisfies the estimate

$$
\left\|J^{0}(t, \tau)\right\| \leq \mathcal{C}^{0} \tau^{-3 / 2}, \quad \tau>0, \quad|t| \leq t^{0},
$$

where $\mathcal{C}^{0}$ is the constant (5.61). 


\section{$\S 6$. The family $A(t)=M^{*} \widehat{A}(t) M$. Approximation OF THE SANDWICHED OPERATOR EXPONENTIAL}

6.1. The operator family $A(t)=M^{*} \widehat{A}(t) M$. As in BSu1, Chapter 1, Subsection 1.5] and [Su2, Subsection 1.3], we consider yet another separable Hilbert space $\widehat{\mathfrak{H}}$ and a family of operators $\hat{X}(t)=\widehat{X}_{0}+t \widehat{X}_{1}: \widehat{\mathfrak{H}} \rightarrow \mathfrak{H}_{*}$ satisfying the assumptions of Subsection 1.1. Let $M: \mathfrak{H} \rightarrow \widehat{\mathfrak{H}}$ be an isomorphism such that $M \operatorname{Dom} X_{0}=\operatorname{Dom} \hat{X}_{0}$ and $X(t)=\hat{X}(t) M:$ $\mathfrak{H} \rightarrow \mathfrak{H}_{*}$. Then

$$
X_{0}=\widehat{X}_{0} M, \quad X_{1}=\widehat{X}_{1} M
$$

In $\widehat{\mathfrak{H}}$, we consider a family of selfadjoint operators $\widehat{A}(t)=\hat{X}(t)^{*} \hat{X}(t)$. Then, obviously,

$$
A(t)=M^{*} \widehat{A}(t) M .
$$

In what follows, all the objects corresponding to the family $\widehat{A}(t)$ will be marked by the upper index "^". For the kernels $\hat{\mathfrak{N}}=\operatorname{Ker} \widehat{X}_{0}$ and $\mathfrak{N}=\operatorname{Ker} X_{0}$ we have $\hat{\mathfrak{N}}=M \mathfrak{N}$. Note that $\widehat{n}=n, \hat{\mathfrak{N}}_{*}=\mathfrak{N}_{*}, \widehat{n}_{*}=n_{*}, \widehat{P}_{*}=P_{*}$.

We denote (cf. [BSu1, Chapter 1, Subsection 5.3], [Su2, Subsection 1.3])

$$
Q:=\left(M M^{*}\right)^{-1}=\left(M^{*}\right)^{-1} M^{-1}: \widehat{\mathfrak{H}} \rightarrow \widehat{\mathfrak{H}} .
$$

The operator $Q$ is positive and continuous together with $Q^{-1}$. In the subspace $\hat{\mathfrak{N}}$, we consider the operators

$$
Q_{\hat{\mathfrak{N}}}=\left.\widehat{P} Q\right|_{\hat{\mathfrak{N}}}, \quad M_{0}=\left(Q_{\hat{\mathfrak{N}}}\right)^{-1 / 2}
$$

As was shown in [Su2, Proposition 1.2], the orthogonal projection $P$ of $\mathfrak{H}$ onto $\mathfrak{N}$ and the orthogonal projection $\hat{P}$ of $\hat{\mathfrak{H}}$ onto $\hat{\mathfrak{N}}$ satisfy the relation

$$
P=M^{-1}\left(Q_{\hat{\mathfrak{N}}}\right)^{-1} \widehat{P}\left(M^{*}\right)^{-1} .
$$

6.2. The operators $\widehat{Z}_{Q}, \widehat{R}, \widehat{S}$, and $\widehat{N}_{Q}$. Let $\widehat{\omega} \in \hat{\mathfrak{N}}$, and let $\widehat{\psi}_{Q} \in \operatorname{Dom} \hat{X}_{0}$ be the solution of the problem

$$
\widehat{X}_{0}^{*}\left(\widehat{X}_{0} \widehat{\psi}_{Q}+\widehat{X}_{1} \widehat{\omega}\right)=0, \quad Q \widehat{\psi}_{Q} \perp \widehat{\mathfrak{N}} .
$$

Equation in (6.6) is understood in the weak sense, cf. (1.4), (1.5). We introduce the operator $\hat{Z}_{Q}: \hat{\mathfrak{H}} \rightarrow \widehat{\mathfrak{H}}$ by the relations

$$
\widehat{Z}_{Q} \widehat{\omega}=\widehat{\psi}_{Q}, \quad \widehat{\omega} \in \hat{\mathfrak{N}} ; \quad \widehat{Z}_{Q} \widehat{x}=0, \quad \widehat{x} \in \hat{\mathfrak{N}}^{\perp} .
$$

By Lemma 6.1 in BSu2, the operator $Z$ defined in Subsection 1.2 and the operator $\widehat{Z}_{Q}$ satisfy

$$
\widehat{Z}_{Q}=M Z M^{-1} \widehat{P} .
$$

The operator $\widehat{R}: \hat{\mathfrak{N}} \rightarrow \mathfrak{N}_{*}$ is defined by analogy with $R$ by the formula $\widehat{R}=\left.P_{*} \widehat{X}_{1}\right|_{\hat{\mathfrak{N}}}$. Note that $\widehat{R} \hat{\omega}=\widehat{X}_{0} \widehat{Z}_{Q} \widehat{\omega}+\hat{X}_{1} \widehat{\omega}, \widehat{\omega} \in \hat{\mathfrak{N}}$. The operators $R$ and $\widehat{R}$ satisfy the identities

$$
R=\left.\widehat{R} M\right|_{\mathfrak{N}}, \quad \widehat{R}=\left.R M^{-1}\right|_{\hat{\mathfrak{N}}} .
$$

The spectral germ $\widehat{S}$ of the family $\widehat{A}(t)$ at $t=0$ is defined as in $(1.10): \widehat{S}=\widehat{R}^{*} \widehat{R}: \hat{\mathfrak{N}} \rightarrow \hat{\mathfrak{N}}$. The germs $S$ and $\widehat{S}$ are related by the formula

$$
S=\left.P M^{*} \widehat{S} M\right|_{\mathfrak{N}}
$$

As a counterpart of the operator $N$ (see (2.28)), we define the operator

$$
\widehat{N}_{Q}=\widehat{Z}_{Q}^{*} \hat{X}_{1}^{*} \widehat{R} \hat{P}+(\widehat{R} \widehat{P})^{*} \widehat{X}_{1} \widehat{Z}_{Q} .
$$


By Lemma 6.2 in BSu2, for the operators $N$ and $\hat{N}_{Q}$ we have

$$
\widehat{N}_{Q}=\widehat{P}\left(M^{*}\right)^{-1} N M^{-1} \widehat{P} \text {. }
$$

6.3. The operators $\widehat{Z}_{2, Q}, \widehat{R}_{2, Q}$, and $\hat{N}_{1, Q}^{0}$. Let $\widehat{\omega} \in \hat{\mathfrak{N}}$. We consider the following problem for $\widehat{\phi}_{Q} \in \operatorname{Dom} \widehat{X}_{0}$ :

$$
\hat{X}_{0}^{*}\left(\widehat{X}_{0} \widehat{\phi}_{Q}+\hat{X}_{1} \hat{Z}_{Q} \widehat{\omega}\right)=-\hat{X}_{1}^{*} \hat{R} \widehat{\omega}+Q\left(Q_{\hat{\mathfrak{N}}}\right)^{-1} \widehat{P} \hat{X}_{1}^{*} \widehat{R} \widehat{\omega}, \quad Q \widehat{\phi}_{Q} \perp \hat{\mathfrak{N}} .
$$

The equation in (6.10) is understood in the weak sense (cf. (1.13)). Clearly, the righthand side of this equation belongs to $\hat{\mathfrak{N}}^{\perp}=\operatorname{Ran} \hat{X}_{0}^{*}$, i.e., the solvability condition is satisfied. We define the operator $\widehat{Z}_{2, Q}: \widehat{\mathfrak{H}} \rightarrow \widehat{\mathfrak{H}}$ by the relations

$$
\widehat{Z}_{2, Q} \widehat{\omega}=\widehat{\phi}_{Q}, \quad \widehat{\omega} \in \hat{\mathfrak{N}} ; \quad \hat{Z}_{2, Q} \widehat{x}=0, \quad \widehat{x} \in \hat{\mathfrak{N}}^{\perp} .
$$

Lemma 6.1. The operator $Z_{2}$ defined in Subsection 1.4 and the operator $\widehat{Z}_{2, Q}$ satisfy

$$
\widehat{Z}_{2, Q}=M Z_{2} M^{-1} \widehat{P} \text {. }
$$

Proof. It suffices to check that $\widehat{Z}_{2, Q} \widehat{\omega}=M Z_{2} M^{-1} \widehat{\omega}, \widehat{\omega} \in \hat{\mathfrak{N}}$. Let $\widehat{\omega} \in \hat{\mathfrak{N}}$, and let $\omega=M^{-1} \widehat{\omega}$. Then $\omega \in \mathfrak{N}$, and by $(6.1),(6.7)$, and (6.8), we have

$$
\widehat{R} \widehat{\omega}=R \omega, \quad \widehat{X}_{1} \widehat{Z}_{Q} \widehat{\omega}=X_{1} Z \omega .
$$

Next, relations (6.1), (6.3), (6.5), and (6.13) imply

$$
\begin{aligned}
M^{*}( & \left.-\widehat{X}_{1}^{*} \widehat{R} \widehat{\omega}+Q\left(Q_{\hat{\mathfrak{N}}}\right)^{-1} \widehat{P} \hat{X}_{1}^{*} \widehat{R} \widehat{\omega}\right) \\
& =-X_{1}^{*} R \omega+M^{-1}\left(Q_{\widehat{\mathfrak{N}}}\right)^{-1} \widehat{P}\left(M^{*}\right)^{-1} X_{1}^{*} R \omega=-X_{1}^{*} R \omega+P X_{1}^{*} R \omega \\
& =-P^{\perp} X_{1}^{*} R \omega .
\end{aligned}
$$

Now, multiplying (6.10) by the operator $M^{*}$ and using (6.1) and (6.13), we conclude that

$$
X_{0}^{*}\left(X_{0} M^{-1} \widehat{\phi}_{Q}+X_{1} Z \omega\right)=-P^{\perp} X_{1}^{*} R \omega .
$$

Due to (6.3), the orthogonality condition in (6.10) yields $\left(M^{*}\right)^{-1} M^{-1} \widehat{\phi}_{Q} \perp \hat{\mathfrak{N}}$, i.e., $\left(M^{-1} \widehat{\phi}_{Q}, M^{-1} \widehat{\zeta}\right)_{\mathfrak{H}}=0, \widehat{\zeta} \in \hat{\mathfrak{N}}$. This is equivalent to the condition $M^{-1} \widehat{\phi}_{Q} \perp \mathfrak{N}$. Comparing this orthogonality condition and equation (6.14) with (1.12), we see that $M^{-1} \widehat{\phi}_{Q}=\phi(\omega)=Z_{2} \omega$. Hence, by (6.11), $\widehat{Z}_{2, Q} \widehat{\omega}=\widehat{\phi}_{Q}=M Z_{2} \omega=M Z_{2} M^{-1} \widehat{\omega}$, $\widehat{\omega} \in \hat{\mathfrak{N}}$.

Now, by analogy with (1.17), we introduce the operator $\widehat{R}_{2, Q}: \hat{\mathfrak{N}} \rightarrow \mathfrak{H}_{*}$ by the relation

$$
\widehat{R}_{2, Q} \widehat{\omega}=\widehat{X}_{0} \widehat{Z}_{2, Q} \widehat{\omega}+\widehat{X}_{1} \widehat{Z}_{Q} \widehat{\omega}, \quad \widehat{\omega} \in \hat{\mathfrak{N}} .
$$

From (1.17) and (6.1), (6.7), (6.12), (6.15) it is seen that the operators $R_{2}$ and $\widehat{R}_{2, Q}$ satisfy the identity $\widehat{R}_{2, Q} \widehat{\omega}=R_{2} \omega$ for $\omega=M^{-1} \widehat{\omega}, \widehat{\omega} \in \hat{\mathfrak{N}}$, i.e.,

$$
R_{2}=\left.\widehat{R}_{2, Q} M\right|_{\mathfrak{N}}, \quad \widehat{R}_{2, Q}=\left.R_{2} M^{-1}\right|_{\hat{\mathfrak{N}}} .
$$

Finally, by analogy with $N_{1}^{0}$ (see (2.49)), we introduce the operator

$$
\widehat{N}_{1, Q}^{0}=\widehat{Z}_{2, Q}^{*} \widehat{X}_{1}^{*} \widehat{R} \hat{P}+(\widehat{R} \widehat{P})^{*} \widehat{X}_{1} \widehat{Z}_{2, Q}+\widehat{R}_{2, Q}^{*} \widehat{R}_{2, Q} \widehat{P}
$$

From $(6.1),(6.8),(6.12)$, and $(6.16)$ we deduce that the operators $N_{1}^{0}$ and $\widehat{N}_{1, Q}^{0}$ satisfy the relation

$$
\widehat{N}_{1, Q}^{0}=\widehat{P}\left(M^{*}\right)^{-1} N_{1}^{0} M^{-1} \widehat{P}
$$


6.4. Approximation of the sandwiched operator exponential. Now our goal is to find an approximation of the operator exponential $e^{-A(t) \tau}$ for the family (6.2) in terms of the characteristics of the operator $\widehat{A}(t)$ and the isomorphism $M$. It turns out (cf. $\mathrm{Su} 2$, Subsection 2.2], $\mathrm{V}, \S 3]$ ) that it is convenient to approximate the sandwiched exponential $M e^{-A(t) \tau} M^{*}$.

From (5.62) it directly follows that

$$
M e^{-A(t) \tau} M^{*}=M e^{-t^{2} S \tau} P M^{*}+\mathcal{K}_{Q}^{(1)}(t, \tau)+\mathcal{K}_{Q}^{(2)}(t, \tau)+M J^{0}(t, \tau) M^{*},
$$

where

$$
\mathcal{K}_{Q}^{(1)}(t, \tau)=M \mathcal{K}^{(1)}(t, \tau) M^{*}, \quad \mathcal{K}_{Q}^{(2)}(t, \tau)=M \mathcal{K}^{(2)}(t, \tau) M^{*} .
$$

As was shown in [Su2, Proposition 2.3], we have

$$
M e^{-t^{2} S \tau} P M^{*}=M_{0} e^{-t^{2} M_{0} \hat{S} M_{0} \tau} M_{0} \widehat{P}=: E_{0}(t, \tau) .
$$

From (5.10) and (6.19) we obtain

$$
\mathcal{K}_{Q}^{(1)}(t, \tau)=t\left(M Z e^{-t^{2} S \tau} P M^{*}+M e^{-t^{2} S \tau} P Z^{*} M^{*}\right)-t^{3} \mathcal{M}_{Q}(t, \tau),
$$

where

$$
\mathcal{M}_{Q}(t, \tau):=\operatorname{MM}(t, \tau) M^{*} .
$$

The following identity is a consequence of (5.11), (6.9), and (6.20):

$$
\mathcal{M}_{Q}(t, \tau)=\int_{0}^{\tau} E_{0}(t, \tau-\rho) \hat{N}_{Q} E_{0}(t, \rho) d \rho .
$$

Now relations (6.21), (6.7), and (6.20) imply

$$
\mathcal{K}_{Q}^{(1)}(t, \tau)=t\left(\widehat{Z}_{Q} E_{0}(t, \tau)+E_{0}(t, \tau) \hat{Z}_{Q}^{*}\right)-t^{3} \mathcal{M}_{Q}(t, \tau) .
$$

Next, by (5.48) and (6.19), we have

$$
\mathcal{K}_{Q}^{(2)}(t, \tau)=\sum_{j=1}^{5} \mathcal{K}_{j, Q}^{(2)}(t, \tau),
$$

where $\mathcal{K}_{j, Q}^{(2)}(t, \tau)=M \mathcal{K}_{j}^{(2)}(t, \tau) M^{*}, \quad j=1, \ldots, 5$.

Taking (6.7), (6.12), and (6.20) into account, from (5.49) we obtain the following representation:

$$
\mathcal{K}_{1, Q}^{(2)}(t, \tau)=t^{2}\left(\widehat{Z}_{2, Q} E_{0}(t, \tau)+E_{0}(t, \tau) \widehat{Z}_{2, Q}^{*}+\widehat{Z}_{Q} E_{0}(t, \tau) \widehat{Z}_{Q}^{*}\right) .
$$

By (6.7) and (6.22), relation (5.50) implies

$$
\mathcal{K}_{2, Q}^{(2)}(t, \tau)=-t^{4}\left(\hat{Z}_{Q} \mathcal{M}_{Q}(t, \tau)+\mathcal{M}_{Q}(t, \tau) \hat{Z}_{Q}^{*}\right) .
$$

Using (6.17) and (6.20), from (5.51) we get

$$
\mathcal{K}_{3, Q}^{(2)}(t, \tau)=-t^{4} \int_{0}^{\tau} E_{0}(t, \tau-\rho) \widehat{N}_{1, Q}^{0} E_{0}(t, \rho) d \rho .
$$

In order to calculate the operator $\mathcal{K}_{4, Q}^{(2)}(t, \tau)$, we use (5.54). Taking (6.20) into account, we have

$$
\mathcal{K}_{4, Q}^{(2)}(t, \tau)=-t^{2} \frac{\partial}{\partial \tau} \int_{0}^{\tau} E_{0}(t, \tau-\rho) \widehat{P}\left(M^{*}\right)^{-1} Z^{*} Z M^{-1} \widehat{P} E_{0}(t, \rho) d \rho .
$$

By (6.7) and (6.3),

$$
\widehat{P}\left(M^{*}\right)^{-1} Z^{*} Z M^{-1} \widehat{P}=\widehat{Z}_{Q}^{*}\left(M^{*}\right)^{-1} M^{-1} \widehat{Z}_{Q}=\widehat{Z}_{Q}^{*} Q \widehat{Z}_{Q}
$$


Consequently,

$$
\mathcal{K}_{4, Q}^{(2)}(t, \tau)=-t^{2} \frac{\partial}{\partial \tau} \int_{0}^{\tau} E_{0}(t, \tau-\rho) \widehat{Z}_{Q}^{*} Q \widehat{Z}_{Q} E_{0}(t, \rho) d \rho .
$$

Finally, combining $(5.53),(6.9),(6.20)$, and (6.22), we arrive at the representation

$$
\mathcal{K}_{5, Q}^{(2)}(t, \tau)=t^{6} \int_{0}^{\tau} E_{0}(t, \tau-\rho) \widehat{N}_{Q} \mathcal{M}_{Q}(t, \rho) d \rho .
$$

Relations (5.12), (5.60), and (6.19) imply the following estimates for the correctors:

$$
\begin{gathered}
\left\|\mathcal{K}_{Q}^{(1)}(t, \tau)\right\|_{\mathfrak{H} \rightarrow \mathfrak{H}} \leq\|M\|^{2}(2 \delta)^{-1 / 2}\left(\left\|X_{1}\right\||t|+\left\|X_{1}\right\|^{3}|t|^{3} \tau\right) e^{-c_{*} t^{2} \tau}, \quad \tau \geq 0, t \in \mathbb{R} \\
\left\|\mathcal{K}_{Q}^{(2)}(t, \tau)\right\|_{\mathfrak{H} \rightarrow \mathfrak{H}} \leq\|M\|^{2}(4 \delta)^{-1}\left(3\left\|X_{1}\right\|^{2} t^{2}+7\left\|X_{1}\right\|^{4} t^{4} \tau+\left\|X_{1}\right\|^{6} t^{6} \tau^{2}\right) e^{-c_{*} t^{2} \tau} \\
\tau \geq 0, t \in \mathbb{R}
\end{gathered}
$$

By (5.63), the error term $M J^{0}(t, \tau) M^{*}=: J_{Q}^{0}(t, \tau)$ in (6.18) satisfies the estimate

$$
\left\|J_{Q}^{0}(t, \tau)\right\|_{\mathfrak{H} \rightarrow \mathfrak{H}} \leq \mathcal{C}^{0}\|M\|^{2} \tau^{-3 / 2}, \quad \tau>0, \quad|t| \leq t^{0} .
$$

We have proved the following theorem.

Theorem 6.2. Let $A(t)$ be an operator of the form (6.2) satisfying the assumptions of Subsection 6.1. Then

$$
M e^{-A(t) \tau} M^{*}=E_{0}(t, \tau)+\mathcal{K}_{Q}^{(1)}(t, \tau)+\mathcal{K}_{Q}^{(2)}(t, \tau)+J_{Q}^{0}(t, \tau),
$$

where the operator $E^{0}(t, \tau)$ is defined by $(6.20), \mathcal{K}_{Q}^{(1)}(t, \tau)$ is defined by (6.23), (6.24), and the operator $\mathcal{K}_{Q}^{(2)}(t, \tau)$ is defined in accordance with (6.25)-(6.30). The operators $\mathcal{K}_{Q}^{(1)}(t, \tau)$ and $\mathcal{K}_{Q}^{(2)}(t, \tau)$ satisfy estimates (6.31) and (6.32), respectively. The error term $J_{Q}^{0}(t, \tau)$ satisfies estimate $(6.33)$, where the constant $\mathcal{C}^{0}$ is given by (5.61).

\section{$\S 7$. Approximation of the Resolvent of the operator $A(t)$}

7.1. Preliminaries. Suppose that condition (5.1) is satisfied. Now our goal is to approximate the resolvent $\left(A(t)+\varepsilon^{2} I\right)^{-1}$ in the operator norm in $\mathfrak{H}$ as $\varepsilon \rightarrow 0$. In [BSu1, the principal term of this approximation was expressed in terms of the resolvent of the operator $t^{2} S$, and an error estimate of order $O\left(\varepsilon^{-1}\right)$ was given. (Note that the resolvent $\left(A(t)+\varepsilon^{2} I\right)^{-1}$ itself is of order $O\left(\varepsilon^{-2}\right)$.) In [BSu2, a more accurate approximation of the resolvent with an error of $O(1)$ was found. The main impact was given by approximation of the operator $\left(A(t)+\varepsilon^{2} I\right)^{-1} F(t)$ in terms of the threshold characteristics of the operator family $A(t)$, while the operator $\left(A(t)+\varepsilon^{2} I\right)^{-1} F(t)^{\perp}$ was "moved to the error". However, it is impossible to obtain an even more accurate approximation of the resolvent $\left(A(t)+\varepsilon^{2} I\right)^{-1}$ in the operator norm in $\mathfrak{H}$ with an error of $O(\varepsilon)$ in the same way. The difficulty is that $\left\|\left(A(t)+\varepsilon^{2} I\right)^{-1} F(t)^{\perp}\right\|=O(1)$, and the operator $\left(A(t)+\varepsilon^{2} I\right)^{-1} F(t)^{\perp}$ "does not move to the error". However, if we multiply the resolvent $\left(A(t)+\varepsilon^{2} I\right)^{-1}$ by a suitable "smoothing" factor (see Subsection 7.5 below), it becomes possible to approximate more accurately.

We denote

$$
\Xi(t, \varepsilon):=\left(t^{2} S+\varepsilon^{2} I_{\mathfrak{N}}\right)^{-1} P .
$$

Relations (5.1) and (5.2) imply the estimates

$$
\begin{array}{rlrl}
\left\|\left(A(t)+\varepsilon^{2} I\right)^{-1}\right\|_{\mathfrak{H} \rightarrow \mathfrak{H}} \leq\left(c_{*} t^{2}+\varepsilon^{2}\right)^{-1}, & & \varepsilon>0, & |t| \leq t^{0}, \\
\|\Xi(t, \varepsilon)\|_{\mathfrak{H} \rightarrow \mathfrak{H}} \leq\left(c_{*} t^{2}+\varepsilon^{2}\right)^{-1}, & & \varepsilon>0, \quad t \in \mathbb{R} .
\end{array}
$$


The operator $\left(A(t)+\varepsilon^{2} I\right)^{-1}$ can be represented as

$$
\left(A(t)+\varepsilon^{2} I\right)^{-1}=\left(A(t)+\varepsilon^{2} I\right)^{-1} F(t)+\left(A(t)+\varepsilon^{2} I\right)^{-1} F(t)^{\perp} .
$$

We start with the analysis of the first term on the right-hand side of (7.4). We shall approximate the operator $\left(A(t)+\varepsilon^{2} I\right)^{-1} F(t)$ with an error term of order $O(|t|)$ (see Theorem 7.1 below).

Denote

$$
G(t, \varepsilon):=\left(A(t)+\varepsilon^{2} I\right)^{-1} F(t)-\Xi(t, \varepsilon) .
$$

The following estimate was proved in [BSu1, Chapter 1, Theorem 5.2] with the help of (4.1) and (4.2):

$$
\|G(t, \varepsilon)\|_{\mathfrak{H} \rightarrow \mathfrak{H}} \leq \mathfrak{C}_{0}|t|\left(c_{*} t^{2}+\varepsilon^{2}\right)^{-1}, \quad \varepsilon>0, \quad|t| \leq t^{0}
$$

Here

$$
\mathfrak{C}_{0}=2 C_{1}+c_{*}^{-1} C_{2}=2 \beta_{1} \delta^{-1 / 2}\left\|X_{1}\right\|+\beta_{2} c_{*}^{-1} \delta^{-1 / 2}\left\|X_{1}\right\|^{3} .
$$

From (7.6) it is seen that $\|G(t, \varepsilon)\|=O\left(\varepsilon^{-1}\right)$ for $|t| \leq t^{0}$.

In BSu2, §5], by using Theorems 4.1 and 4.2, a more accurate approximation of the operator (7.5) was obtained:

$$
G(t, \varepsilon)=\mathfrak{K}^{(1)}(t, \varepsilon)+\mathcal{J}(t, \varepsilon) .
$$

Here the corrector $\mathfrak{K}^{(1)}(t, \varepsilon)$ is given by

$$
\mathfrak{K}^{(1)}(t, \varepsilon)=t\left(Z \Xi(t, \varepsilon)+\Xi(t, \varepsilon) Z^{*}\right)-t^{3} \Xi(t, \varepsilon) N \Xi(t, \varepsilon),
$$

the operator $Z$ is defined in Subsection 1.2, and $N$ is defined by (2.28). Relations (1.7), (2.29), and (7.3) imply the following estimate for the operator (7.9):

$$
\begin{aligned}
& \left\|\mathfrak{K}^{(1)}(t, \varepsilon)\right\|_{\mathfrak{H} \rightarrow \mathfrak{H}} \leq 2|t|\|Z\|\|\Xi(t, \varepsilon)\|+|t|^{3}\|N\|\|\Xi(t, \varepsilon)\|^{2} \\
& \quad \leq(2 \delta)^{-1 / 2}\left(|t|\left\|X_{1}\right\|\left(c_{*} t^{2}+\varepsilon^{2}\right)^{-1}+|t|^{3}\left\|X_{1}\right\|^{3}\left(c_{*} t^{2}+\varepsilon^{2}\right)^{-2}\right), \quad \varepsilon>0, \quad t \in \mathbb{R} .
\end{aligned}
$$

We see that $\left\|\mathfrak{K}^{(1)}(t, \varepsilon)\right\|=O\left(\varepsilon^{-1}\right)$.

As was proved in [BSu2, $\S 5]$, the error term $\mathcal{J}(t, \varepsilon)$ satisfies

$$
\|\mathcal{J}(t, \varepsilon)\|_{\mathfrak{H} \rightarrow \mathfrak{H}} \leq \mathfrak{C}_{1}, \quad \varepsilon>0, \quad|t| \leq t^{0},
$$

where

$$
\mathfrak{C}_{1}=\delta^{-1}\left(\beta^{(1)} c_{*}^{-1}\left\|X_{1}\right\|^{2}+\beta^{(2)} c_{*}^{-2}\left\|X_{1}\right\|^{4}+\beta^{(3)} c_{*}^{-3}\left\|X_{1}\right\|^{6}\right) .
$$

7.2. The analysis of the operators $F(t)^{\perp} \Xi(t, \varepsilon)$ and $F(t)\left(A(t)+\varepsilon^{2} I\right)^{-1} P^{\perp}$. The operator (7.5) can be represented as

$$
G(t, \varepsilon)=F(t)\left(A(t)+\varepsilon^{2} I\right)^{-1} P^{\perp}-F(t)^{\perp} \Xi(t, \varepsilon)+G_{0}(t, \varepsilon),
$$

where

$$
G_{0}(t, \varepsilon):=F(t)\left(A(t)+\varepsilon^{2} I\right)^{-1} P-F(t) \Xi(t, \varepsilon) .
$$

We start with the analysis of the second term on the right-hand side of (7.13). By (2.52), we have $F(t)^{\perp} P=(P-F(t)) P=-\left(t F_{1}+t^{2} F_{2}+F_{3}(t)\right) P$. Hence,

$$
-F(t)^{\perp} \Xi(t, \varepsilon)=\left(t F_{1}+t^{2} F_{2}\right) \Xi(t, \varepsilon)+F_{3}(t) \Xi(t, \varepsilon) .
$$

Relations (4.9) and (7.3) imply the following estimate for the last term on the right-hand side of (7.15):

$$
\left\|F_{3}(t) \Xi(t, \varepsilon)\right\|_{\mathfrak{H} \rightarrow \mathfrak{H}} \leq C_{5}|t|^{3}\left(c_{*} t^{2}+\varepsilon^{2}\right)^{-1} \leq C_{5} c_{*}^{-1}|t|, \quad \varepsilon>0, \quad|t| \leq t^{0} .
$$

Taking (5.28) into account, we transform the first term on the right-hand side of (7.15):

$$
\left(t F_{1}+t^{2} F_{2}\right) \Xi(t, \varepsilon)=\left(t Z+t^{2} Z_{2}-t^{2} Z^{*} Z\right) \Xi(t, \varepsilon) .
$$


Consider the operator $F(t)\left(A(t)+\varepsilon^{2} I\right)^{-1} P^{\perp}$. By (2.52),

$$
F(t) P^{\perp}=F(t)(F(t)-P)=F(t)\left(t F_{1}+t^{2} F_{2}+F_{3}(t)\right) .
$$

Hence,

$$
F(t)\left(A(t)+\varepsilon^{2} I\right)^{-1} P^{\perp}=\left(A(t)+\varepsilon^{2} I\right)^{-1} F(t)\left(t F_{1}+t^{2} F_{2}+F_{3}(t)\right) .
$$

From (4.9) and (7.2) it follows that

$$
\begin{array}{r}
\left\|\left(A(t)+\varepsilon^{2} I\right)^{-1} F(t) F_{3}(t)\right\|_{\mathfrak{H} \rightarrow \mathfrak{H}} \leq C_{5}|t|^{3}\left(c_{*} t^{2}+\varepsilon^{2}\right)^{-1} \leq C_{5} c_{*}^{-1}|t|, \\
\varepsilon>0, \quad|t| \leq t^{0} .
\end{array}
$$

Next, using the notation (7.5), we can write

$$
\left(A(t)+\varepsilon^{2} I\right)^{-1} F(t) t^{2} F_{2}=\Xi(t, \varepsilon) t^{2} F_{2}+G(t, \varepsilon) t^{2} F_{2} .
$$

Relations (7.6) and (2.14) imply the inequality

$$
\left\|G(t, \varepsilon) t^{2} F_{2}\right\|_{\mathfrak{H} \rightarrow \mathfrak{H}} \leq \mathfrak{C}_{2}|t|^{3}\left(c_{*} t^{2}+\varepsilon^{2}\right)^{-1} \leq \mathfrak{C}_{2} c_{*}^{-1}|t|, \quad \varepsilon>0, \quad|t| \leq t^{0},
$$

where

$$
\mathfrak{C}_{2}=3 \mathfrak{C}_{0}\left\|X_{1}\right\|^{2}(4 \delta)^{-1}
$$

By (5.28), we have $P F_{2}=Z_{2}^{*}-Z^{*} Z$. Consequently,

$$
\Xi(t, \varepsilon) t^{2} F_{2}=t^{2} \Xi(t, \varepsilon)\left(Z_{2}^{*}-Z^{*} Z\right) .
$$

Finally, we consider the term $\left(A(t)+\varepsilon^{2} I\right)^{-1} F(t) t F_{1}$. By (7.5) and (7.8),

$$
\left(A(t)+\varepsilon^{2} I\right)^{-1} F(t) t F_{1}=\left(\Xi(t, \varepsilon)+\mathfrak{K}^{(1)}(t, \varepsilon)\right) t F_{1}+\mathcal{J}(t, \varepsilon) t F_{1} .
$$

The estimate for the last term on the right-hand side of (7.24) is a consequence of (7.11) and (2.7):

$$
\left\|\mathcal{J}(t, \varepsilon) t F_{1}\right\|_{\mathfrak{H} \rightarrow \mathfrak{H}} \leq \mathfrak{C}_{3}|t|, \quad \varepsilon>0, \quad|t| \leq t^{0}
$$

where

$$
\mathfrak{C}_{3}=\mathfrak{C}_{1}\left\|X_{1}\right\|(2 \delta)^{-1 / 2}
$$

Using (7.9) and the identities $P F_{1}=Z^{*}, Z^{*} F_{1}=Z^{*} Z$ (see (5.28)), we obtain the following representation for the first term on the right-hand side of (7.24):

$$
\begin{aligned}
& \left(\Xi(t, \varepsilon)+\mathfrak{K}^{(1)}(t, \varepsilon)\right) t F_{1} \\
& \quad=t \Xi(t, \varepsilon) Z^{*}+t^{2} Z \Xi(t, \varepsilon) Z^{*}+t^{2} \Xi(t, \varepsilon) Z^{*} Z-t^{4} \Xi(t, \varepsilon) N \Xi(t, \varepsilon) Z^{*} .
\end{aligned}
$$

Now we summarize the study of the terms $F(t)^{\perp} \Xi(t, \varepsilon)$ and $F(t)\left(A(t)+\varepsilon^{2} I\right)^{-1} P^{\perp}$. Relations (7.15), (7.17), (7.18), (7.20), (7.23), (7.24), and (7.27) imply the following representation:

$$
\begin{aligned}
& F(t)\left(A(t)+\varepsilon^{2} I\right)^{-1} P^{\perp}-F(t)^{\perp} \Xi(t, \varepsilon) \\
& =t\left(Z \Xi+\Xi Z^{*}\right)+t^{2}\left(Z_{2} \Xi+\Xi Z_{2}^{*}\right)-t^{2} Z^{*} Z \Xi+t^{2} Z \Xi Z^{*}-t^{4} \Xi N \Xi Z^{*}+\mathcal{J}_{1}(t, \varepsilon),
\end{aligned}
$$

where $\Xi=\Xi(t, \varepsilon)$ and

$$
\mathcal{J}_{1}(t, \varepsilon)=F_{3}(t) \Xi(t, \varepsilon)+\left(A(t)+\varepsilon^{2} I\right)^{-1} F(t) F_{3}(t)+G(t, \varepsilon) t^{2} F_{2}+\mathcal{J}(t, \varepsilon) t F_{1} .
$$

By (7.16), (7.19), (7.21), and (7.25), we obtain the following estimate of the error term:

$$
\left\|\mathcal{J}_{1}(t, \varepsilon)\right\|_{\mathfrak{H} \rightarrow \mathfrak{H}} \leq\left(2 C_{5} c_{*}^{-1}+\mathfrak{C}_{2} c_{*}^{-1}+\mathfrak{C}_{3}\right)|t|, \quad \varepsilon>0, \quad|t| \leq t^{0} .
$$


7.3. The study of the operator $G_{0}(t, \varepsilon)$. The operator (7.14) satisfies the identity

$$
G_{0}(t, \varepsilon)=-F(t)\left(A(t)+\varepsilon^{2} I\right)^{-1}\left(A(t) F(t)-t^{2} S P\right) \Xi(t, \varepsilon) .
$$

Combining this with (2.53), we get

$$
G_{0}(t, \varepsilon)=-F(t)\left(A(t)+\varepsilon^{2} I\right)^{-1}\left(t^{3} K+t^{4} W+\Psi(t)\right) \Xi(t, \varepsilon) .
$$

From (4.16) and (7.2), (7.3) it follows that

$$
\begin{array}{r}
\left\|F(t)\left(A(t)+\varepsilon^{2} I\right)^{-1} \Psi(t) \Xi(t, \varepsilon)\right\|_{\mathfrak{H} \rightarrow \mathfrak{H}} \leq C_{6}|t|^{5}\left(c_{*} t^{2}+\varepsilon^{2}\right)^{-2} \leq C_{6} c_{*}^{-2}|t|, \\
\varepsilon>0, \quad|t| \leq t^{0} .
\end{array}
$$

Next, taking the notation (7.5) into account, we have the relation

$$
F(t)\left(A(t)+\varepsilon^{2} I\right)^{-1} t^{4} W \Xi(t, \varepsilon)=t^{4} \Xi(t, \varepsilon) W \Xi(t, \varepsilon)+t^{4} G(t, \varepsilon) W \Xi(t, \varepsilon) .
$$

From (2.51), (7.3), and (7.6) it follows that

$$
\left\|t^{4} G(t, \varepsilon) W \Xi(t, \varepsilon)\right\|_{\mathfrak{H} \rightarrow \mathfrak{H}} \leq \mathfrak{C}_{4}|t|^{5}\left(c_{*} t^{2}+\varepsilon^{2}\right)^{-2} \leq \mathfrak{C}_{4} c_{*}^{-2}|t|, \quad \varepsilon>0, \quad|t| \leq t^{0},
$$

where

$$
\mathfrak{C}_{4}=5 \mathfrak{C}_{0}(2 \delta)^{-1}\left\|X_{1}\right\|^{4}
$$

By (5.45), the first term on the right-hand side of (7.32) is represented as

$$
t^{4} \Xi(t, \varepsilon) W \Xi(t, \varepsilon)=t^{4} \Xi(t, \varepsilon)\left(N_{1}^{0}-Z^{*} Z S P-S P Z^{*} Z\right) \Xi(t, \varepsilon) .
$$

Now we consider the term involving $K$ in (7.30). By (7.5) and (7.8),

$$
F(t)\left(A(t)+\varepsilon^{2} I\right)^{-1} t^{3} K \Xi(t, \varepsilon)=\left(\Xi(t, \varepsilon)+\mathfrak{K}^{(1)}(t, \varepsilon)+\mathcal{J}(t, \varepsilon)\right) t^{3} K \Xi(t, \varepsilon) .
$$

Relations (2.30), (7.3), and (7.11) imply the estimate

$$
\left\|\mathcal{J}(t, \varepsilon) t^{3} K \Xi(t, \varepsilon)\right\|_{\mathfrak{H} \rightarrow \mathfrak{H}} \leq \mathfrak{C}_{5}|t|^{3}\left(c_{*} t^{2}+\varepsilon^{2}\right)^{-1} \leq \mathfrak{C}_{5} c_{*}^{-1}|t|, \quad \varepsilon>0, \quad|t| \leq t^{0},
$$

where

$$
\mathfrak{C}_{5}=\mathfrak{C}_{1} 2(2 \delta)^{-1 / 2}\left\|X_{1}\right\|^{3}
$$

By (7.9), we have

$$
\left(\Xi+\mathfrak{K}^{(1)}(t, \varepsilon)\right) t^{3} K \Xi=t^{3} \Xi K \Xi+t^{4} Z \Xi K \Xi+t^{4} \Xi Z^{*} K \Xi-t^{6} \Xi N \Xi K \Xi .
$$

We apply identities (5.44) and observe that

$$
Z^{*} K=Z^{*} K_{0}=Z^{*} Z S P
$$

which follows from (2.19), (2.22) and the properties of the operators $N$ and $Z$. Then the operator (7.39) takes the form

$$
\left(\Xi+\mathfrak{K}^{(1)}(t, \varepsilon)\right) t^{3} K \Xi=t^{3} \Xi N \Xi+t^{4} Z \Xi N \Xi+t^{4} \Xi Z^{*} Z S P \Xi-t^{6} \Xi N \Xi N \Xi .
$$

As a result, relations (7.30), (7.32), (7.35), (7.36), and (7.40) imply the following representation for the operator (7.14):

(7.41) $G_{0}(t, \varepsilon)=-t^{3} \Xi N \Xi-t^{4} \Xi N_{1}^{0} \Xi-t^{4} Z \Xi N \Xi+t^{4} \Xi S P Z^{*} Z \Xi+t^{6} \Xi N \Xi N \Xi+\mathcal{J}_{2}(t, \varepsilon)$, where $\Xi=\Xi(t, \varepsilon)$ and

$$
\mathcal{J}_{2}(t, \varepsilon)=-F(t)\left(A(t)+\varepsilon^{2} I\right)^{-1} \Psi(t) \Xi(t, \varepsilon)-t^{4} G(t, \varepsilon) W \Xi(t, \varepsilon)-\mathcal{J}(t, \varepsilon) t^{3} K \Xi(t, \varepsilon) .
$$

From (7.31), (7.33), and (7.37) we obtain the following estimate of the error term:

$$
\left\|\mathcal{J}_{2}(t, \varepsilon)\right\|_{\mathfrak{H} \rightarrow \mathfrak{H}} \leq\left(C_{6} c_{*}^{-2}+\mathfrak{C}_{4} c_{*}^{-2}+\mathfrak{C}_{5} c_{*}^{-1}\right)|t|, \quad \varepsilon>0, \quad|t| \leq t^{0} .
$$


7.4. Approximation of the operator $\left(A(t)+\varepsilon^{2} I\right)^{-1} F(t)$. Now relations (7.5), (7.13), (7.28), and (7.41) imply the representation

$$
\left(A(t)+\varepsilon^{2} I\right)^{-1} F(t)=\Xi(t, \varepsilon)+\mathfrak{K}^{(1)}(t, \varepsilon)+\mathfrak{K}^{(2)}(t, \varepsilon)+\mathcal{J}_{1}(t, \varepsilon)+\mathcal{J}_{2}(t, \varepsilon),
$$

where the first corrector $\mathfrak{K}^{(1)}(t, \varepsilon)$ is defined by $(7.9)$, and the second corrector $\mathfrak{K}^{(2)}(t, \varepsilon)$ is the sum of five terms:

$$
\mathfrak{K}^{(2)}(t, \varepsilon)=\sum_{j=1}^{5} \mathfrak{K}_{j}^{(2)}(t, \varepsilon) .
$$

Here

$$
\begin{aligned}
& \mathfrak{K}_{1}^{(2)}(t, \varepsilon)=t^{2}\left(Z_{2} \Xi(t, \varepsilon)+\Xi(t, \varepsilon) Z_{2}^{*}+Z \Xi(t, \varepsilon) Z^{*}\right), \\
& \mathfrak{K}_{2}^{(2)}(t, \varepsilon)=-t^{4}\left(Z \Xi(t, \varepsilon) N \Xi(t, \varepsilon)+\Xi(t, \varepsilon) N \Xi(t, \varepsilon) Z^{*}\right), \\
& \mathfrak{K}_{3}^{(2)}(t, \varepsilon)=-t^{4} \Xi(t, \varepsilon) N_{1}^{0} \Xi(t, \varepsilon), \\
& \mathfrak{K}_{4}^{(2)}(t, \varepsilon)=-t^{2} Z^{*} Z \Xi(t, \varepsilon)+t^{4} \Xi(t, \varepsilon) S P Z^{*} Z \Xi(t, \varepsilon)=-t^{2} \varepsilon^{2} \Xi(t, \varepsilon) Z^{*} Z \Xi(t, \varepsilon),
\end{aligned}
$$

We estimate the operators (7.45)-(7.49). From (1.7), (1.16), and (7.3) it follows that

$$
\left\|\mathfrak{K}_{1}^{(2)}(t, \varepsilon)\right\|_{\mathfrak{H} \rightarrow \mathfrak{H}} \leq 5(8 \delta)^{-1}\left\|X_{1}\right\|^{2} t^{2}\left(c_{*} t^{2}+\varepsilon^{2}\right)^{-1}, \quad \varepsilon>0, \quad t \in \mathbb{R} .
$$

By (1.7), (2.29), and (7.3), we have

$$
\left\|\mathfrak{K}_{2}^{(2)}(t, \varepsilon)\right\|_{\mathfrak{H} \rightarrow \mathfrak{H}} \leq(2 \delta)^{-1}\left\|X_{1}\right\|^{4} t^{4}\left(c_{*} t^{2}+\varepsilon^{2}\right)^{-2}, \quad \varepsilon>0, \quad t \in \mathbb{R} .
$$

Relations (2.50) and (7.3) imply

$$
\left\|\mathfrak{K}_{3}^{(2)}(t, \varepsilon)\right\|_{\mathfrak{H} \rightarrow \mathfrak{H}} \leq 9(8 \delta)^{-1}\left\|X_{1}\right\|^{4} t^{4}\left(c_{*} t^{2}+\varepsilon^{2}\right)^{-2}, \quad \varepsilon>0, \quad t \in \mathbb{R} .
$$

Applying (1.7) and (7.3), we obtain

$$
\left\|\mathfrak{K}_{4}^{(2)}(t, \varepsilon)\right\|_{\mathfrak{H} \rightarrow \mathfrak{H}} \leq(8 \delta)^{-1}\left\|X_{1}\right\|^{2} t^{2} \varepsilon^{2}\left(c_{*} t^{2}+\varepsilon^{2}\right)^{-2}, \quad \varepsilon>0, \quad t \in \mathbb{R} .
$$

Finally, from (2.29) and (7.3) it follows that

$$
\left\|\mathfrak{K}_{5}^{(2)}(t, \varepsilon)\right\|_{\mathfrak{H} \rightarrow \mathfrak{H}} \leq(2 \delta)^{-1}\left\|X_{1}\right\|^{6} t^{6}\left(c_{*} t^{2}+\varepsilon^{2}\right)^{-3}, \quad \varepsilon>0, \quad t \in \mathbb{R} .
$$

As a result, relations (7.50)-(7.54) yield the following estimate for the operator (7.44):

$$
\begin{aligned}
\left\|\mathfrak{K}^{(2)}(t, \varepsilon)\right\|_{\mathfrak{H} \rightarrow \mathfrak{H}} & \leq(8 \delta)^{-1}\left(5\left\|X_{1}\right\|^{2} t^{2}\left(c_{*} t^{2}+\varepsilon^{2}\right)^{-1}+13\left\|X_{1}\right\|^{4} t^{4}\left(c_{*} t^{2}+\varepsilon^{2}\right)^{-2}\right. \\
& \left.+\left\|X_{1}\right\|^{2} t^{2} \varepsilon^{2}\left(c_{*} t^{2}+\varepsilon^{2}\right)^{-2}+4\left\|X_{1}\right\|^{6} t^{6}\left(c_{*} t^{2}+\varepsilon^{2}\right)^{-3}\right), \quad \varepsilon>0, \quad t \in \mathbb{R} .
\end{aligned}
$$

The error terms in (7.43) satisfy estimates (7.29) and (7.42). We denote $\mathcal{J}^{0}(t, \varepsilon)=$ $\mathcal{J}_{1}(t, \varepsilon)+\mathcal{J}_{2}(t, \varepsilon)$. Then

$$
\left\|\mathcal{J}^{0}(t, \varepsilon)\right\|_{\mathfrak{H} \rightarrow \mathfrak{H}} \leq \mathfrak{C}^{0}|t|, \quad \varepsilon>0, \quad|t| \leq t^{0},
$$

where $\mathfrak{C}^{0}=2 C_{5} c_{*}^{-1}+\mathfrak{C}_{2} c_{*}^{-1}+\mathfrak{C}_{3}+C_{6} c_{*}^{-2}+\mathfrak{C}_{4} c_{*}^{-2}+\mathfrak{C}_{5} c_{*}^{-1}$. From (4.10), (4.17), (7.7), (7.12), (7.22), (7.26), (7.34), and (7.38) we see that the constant $\mathfrak{C}^{0}$ is given by

$$
\mathfrak{C}^{0}=\delta^{-3 / 2}\left(\breve{\beta}_{1} c_{*}^{-1}\left\|X_{1}\right\|^{3}+\check{\beta}_{2} c_{*}^{-2}\left\|X_{1}\right\|^{5}+\check{\beta}_{3} c_{*}^{-3}\left\|X_{1}\right\|^{7}+\check{\beta}_{4} c_{*}^{-4}\left\|X_{1}\right\|^{9}\right),
$$

where $\breve{\beta}_{j}, j=1,2,3,4$, are some absolute constants.

We have proved the following result. 
Theorem 7.1. We have

$$
\left(A(t)+\varepsilon^{2} I\right)^{-1} F(t)=\Xi(t, \varepsilon)+\mathfrak{K}^{(1)}(t, \varepsilon)+\mathfrak{K}^{(2)}(t, \varepsilon)+\mathcal{J}^{0}(t, \varepsilon),
$$

where the operator $\Xi(t, \varepsilon)$ is defined by $(7.1), \mathfrak{K}^{(1)}(t, \varepsilon)$ is defined by $(7.9)$, and the operator $\mathfrak{K}^{(2)}(t, \varepsilon)$ is defined in accordance with $(7.44)-(7.49)$. The operators $\mathfrak{K}^{(1)}(t, \varepsilon)$ and $\mathfrak{K}^{(2)}(t, \varepsilon)$ satisfy estimates (7.10) and (7.55), respectively. The error term $\mathcal{J}^{0}(t, \varepsilon)$ satisfies estimate (7.56), where $\mathfrak{C}^{0}$ is the constant (7.57).

7.5. Approximation of the "smoothed" resolvent. Let $\breve{A}(t)$ be an (auxiliary) selfadjoint operator in $\mathfrak{H}$ admitting a factorization of the form $(1.1)$, i.e., $\breve{A}(t)=\breve{X}(t)^{*} \breve{X}(t)$, $\breve{X}(t)=\check{X}_{0}+t \breve{X}_{1}$. Assume that $\breve{A}(t)$ satisfies the same assumptions as $A(t)$ (see Subsection 1.1), and that $\operatorname{Ker} \breve{A}(0)=\operatorname{Ker} A(0)=\mathfrak{N}$. Let $\breve{d}^{0}$ be the distance from the point $\lambda_{0}=0$ to the rest of the spectrum of the operator $\breve{A}(0)$. We fix a number $\breve{\delta} \in\left(0, \breve{d}^{0} / 8\right)$ and put $\breve{t}^{0}=\breve{\delta}^{1 / 2}\left\|\breve{X}_{1}\right\|^{-1}$. Let $\breve{F}(t)$ be the spectral projection of the operator $\breve{A}(t)$ for the interval $[0, \check{\delta}]$. Applying Theorem 4.1 to the operator $\breve{A}(t)$, we have

$$
\|\breve{F}(t)-P\|_{\mathfrak{H} \rightarrow \mathfrak{H}} \leq \check{C}_{1}|t|, \quad|t| \leq \breve{t}^{0},
$$

where $\check{C}_{1}=\beta_{1} \check{\delta}^{-1 / 2}\left\|\check{X}_{1}\right\|$.

Next, assume that $\breve{A}(t)$ satisfies a condition of the form (5.1):

$$
\check{A}(t) \geq \check{c}_{*} t^{2} I, \quad|t| \leq \check{t}^{0}, \quad \check{c}_{*}>0 .
$$

Then

$$
\left\|\left(\breve{A}(t)+\varepsilon^{2} I\right)^{-1 / 2}\right\|_{\mathfrak{H} \rightarrow \mathfrak{H}} \leq\left(\breve{c}_{*} t^{2}+\varepsilon^{2}\right)^{-1 / 2}, \quad|t| \leq \breve{t}^{0} .
$$

Obviously,

$$
\left\|\breve{F}(t)^{\perp}\left(\breve{A}(t)+\varepsilon^{2} I\right)^{-1 / 2}\right\|_{\mathfrak{H} \rightarrow \mathfrak{H}} \leq \breve{\delta}^{-1 / 2}, \quad|t| \leq \breve{t}^{0} .
$$

From (7.59), (7.61), and (7.62) it follows that

$$
\left\|P^{\perp}\left(\breve{A}(t)+\varepsilon^{2} I\right)^{-1 / 2}\right\|_{\mathfrak{H} \rightarrow \mathfrak{H}} \leq \check{\delta}^{-1 / 2}+\check{C}_{1}|t|\left(\check{C}_{*} t^{2}+\varepsilon^{2}\right)^{-1 / 2} \leq \check{C}_{2}, \quad|t| \leq \breve{t}^{0},
$$

where

$$
\breve{C}_{2}=\breve{\delta}^{-1 / 2}+\breve{C}_{1} \check{c}_{*}^{-1 / 2}=\check{\delta}^{-1 / 2}\left(1+\beta_{1}\left\|\breve{X}_{1}\right\| \check{c}_{*}^{-1 / 2}\right) .
$$

We start with (7.4) and (7.58). Multiplying by the operator $\varepsilon\left(\check{A}(t)+\varepsilon^{2} I\right)^{-1 / 2}$ from the right, we obtain

$$
\begin{aligned}
& \left(\left(A(t)+\varepsilon^{2} I\right)^{-1}-\Xi(t, \varepsilon)-\mathfrak{K}^{(1)}(t, \varepsilon)-\mathfrak{K}^{(2)}(t, \varepsilon)\right) \varepsilon\left(\breve{A}(t)+\varepsilon^{2} I\right)^{-1 / 2} \\
& \quad=\mathcal{J}^{0}(t, \varepsilon) \varepsilon\left(\breve{A}(t)+\varepsilon^{2} I\right)^{-1 / 2}+\left(A(t)+\varepsilon^{2} I\right)^{-1} F(t)^{\perp} \varepsilon\left(\breve{A}(t)+\varepsilon^{2} I\right)^{-1 / 2} .
\end{aligned}
$$

The first term on the right-hand side of (7.65) is estimated with the help of (7.56) and (7.61):

$$
\begin{array}{r}
\left\|\mathcal{J}^{0}(t, \varepsilon) \varepsilon\left(\breve{A}(t)+\varepsilon^{2} I\right)^{-1 / 2}\right\|_{\mathfrak{H} \rightarrow \mathfrak{H}} \leq \mathfrak{C}^{0}|t| \varepsilon\left(\check{c}_{*} t^{2}+\varepsilon^{2}\right)^{-1 / 2} \leq \mathfrak{C}^{0}\left(\breve{c}_{*}\right)^{-1 / 2} \varepsilon \\
|t| \leq \min \left\{t^{0}, \breve{t}^{0}\right\}, \quad \varepsilon>0 .
\end{array}
$$

In order to estimate the second term on the right-hand side of (7.65), we use the inequality

$$
\left\|\left(A(t)+\varepsilon^{2} I\right)^{-1} F(t)^{\perp}\right\|_{\mathfrak{H} \rightarrow \mathfrak{H}} \leq(3 \delta)^{-1}, \quad|t| \leq t^{0}, \quad \varepsilon>0,
$$

which follows from (1.2). Then

$$
\begin{aligned}
\|(A(t) & \left.+\varepsilon^{2} I\right)^{-1} F(t)^{\perp} \varepsilon\left(\breve{A}(t)+\varepsilon^{2} I\right)^{-1 / 2} \|_{\mathfrak{H} \rightarrow \mathfrak{H}} \\
\leq & \leq(3 \delta)^{-1} \varepsilon\left\|F(t)^{\perp}\left(\check{A}(t)+\varepsilon^{2} I\right)^{-1 / 2}\right\|_{\mathfrak{H} \rightarrow \mathfrak{H}}, \quad|t| \leq t^{0}, \quad \varepsilon>0 .
\end{aligned}
$$


Next,

$$
\begin{aligned}
& \left\|F(t)^{\perp}\left(\check{A}(t)+\varepsilon^{2} I\right)^{-1 / 2}\right\|_{\mathfrak{H} \rightarrow \mathfrak{H}} \\
& \quad \leq\left\|(P-F(t))\left(\check{A}(t)+\varepsilon^{2} I\right)^{-1 / 2}\right\|_{\mathfrak{H} \rightarrow \mathfrak{H}}+\left\|P^{\perp}\left(\check{A}(t)+\varepsilon^{2} I\right)^{-1 / 2}\right\|_{\mathfrak{H} \rightarrow \mathfrak{H}} .
\end{aligned}
$$

Combining this with (4.1), (7.61), and (7.63), we obtain

$$
\begin{array}{r}
\left\|F(t)^{\perp}\left(\check{A}(t)+\varepsilon^{2} I\right)^{-1 / 2}\right\|_{\mathfrak{H} \rightarrow \mathfrak{H}} \leq C_{1}|t|\left(\check{c}_{*} t^{2}+\varepsilon^{2}\right)^{-1 / 2}+\check{C}_{2} \leq C_{1}\left(\check{c}_{*}\right)^{-1 / 2}+\check{C}_{2}, \\
|t| \leq \min \left\{t^{0}, \breve{t}^{0}\right\}, \quad \varepsilon>0 .
\end{array}
$$

From (7.68) and (7.69) it follows that

$$
\begin{array}{r}
\left\|\left(A(t)+\varepsilon^{2} I\right)^{-1} F(t)^{\perp} \varepsilon\left(\breve{A}(t)+\varepsilon^{2} I\right)^{-1 / 2}\right\|_{\mathfrak{H} \rightarrow \mathfrak{H} \leq} \leq(3 \delta)^{-1}\left(C_{1}\left(\check{c}_{*}\right)^{-1 / 2}+\check{C}_{2}\right) \varepsilon, \\
|t| \leq \min \left\{t^{0}, \breve{t}^{0}\right\}, \quad \varepsilon>0 .
\end{array}
$$

We denote

$$
\widetilde{\mathfrak{C}}=\mathfrak{C}^{0}\left(\breve{c}_{*}\right)^{-1 / 2}+(3 \delta)^{-1}\left(C_{1}\left(\check{c}_{*}\right)^{-1 / 2}+\check{C}_{2}\right),
$$

where the constant $\mathfrak{C}^{0}$ is defined by $(7.57), C_{1}$ is defined by $(4.3)$, and $\breve{C}_{2}$ is given by (7.64). Now the representation (7.65) and estimates (7.66) and (7.70) directly imply the following result.

Theorem 7.2. Under the assumptions of Theorem 7.1, let $\breve{A}(t)$ be an operator satisfying the conditions described above. Then for $|t| \leq \min \left\{t^{0}, \breve{t}^{0}\right\}$ and $\varepsilon>0$ we have

$$
\left\|\left(\left(A(t)+\varepsilon^{2} I\right)^{-1}-\Xi(t, \varepsilon)-\mathfrak{K}^{(1)}(t, \varepsilon)-\mathfrak{K}^{(2)}(t, \varepsilon)\right) \varepsilon\left(\check{A}(t)+\varepsilon^{2} I\right)^{-1 / 2}\right\|_{\mathfrak{H} \rightarrow \mathfrak{H}} \leq \widetilde{\mathfrak{C}} \varepsilon .
$$

The constant $\widetilde{\mathfrak{C}}$ is defined by (7.71) and depends only on $\delta,\left\|X_{1}\right\|, c_{*}, \check{\delta},\left\|\check{X}_{1}\right\|$, and $\check{c}_{*}$.

Remark 7.3. One could take $A(t)$ itself for the role of $\breve{A}(t)$. However, this is inconvenient for applications to differential operators. In applications, the role of $A(t)$ is played by a differential operator with variable coefficients (depending on the parameter $t$ ), while it is convenient to choose $\breve{A}(t)$ to be a differential operator with constant coefficients. Herewith, the operator $\varepsilon\left(\breve{A}(t)+\varepsilon^{2} I\right)^{-1 / 2}$ is smoothing indeed. Therefore, we transfer the term "smoothing operator" to the abstract setting.

\section{REFERENCES}

[BSu1] M. Sh. Birman and T. A. Suslina, Second order periodic differential operators. Threshold properties and homogenization, Algebra i Analiz 15 (2003), no. 5, 1-108; English transl., St. Petersburg Math. J. 15 (2004), no. 5, 639-714. MR2068790 (2005k:47097)

[BSu2] _ Threshold approximations with corrector for the resolvent of a factorized selfadjoint operator family, Algebra i Analiz 17 (2005), no. 5, 69-90; English transl., St. Petersburg Math. J. 17 (2006), no. 5, 745-762. MR2241423 (2008d:47047)

[BSu3] _ Homogenization with corrector term for periodic elliptic differential operators, Algebra i Analiz 17 (2005), no. 6, 1-104; English transl., St. Petersburg Math. J. 17 (2006), no. 6, 897-973. MR2202045 (2006k:35011)

[BSu4] _ Homogenization with corrector for periodic differential operators. Approximation of solutions in the Sobolev class $H^{1}\left(\mathbb{R}^{d}\right)$, Algebra i Analiz 18 (2006), no. 6, 1-130; English transl., St. Petersburg Math. J. 18 (2007), no. 6, 857-955. MR2307356 (2008d:35008)

[V] E. S. Vasilevskaya, A periodic parabolic Cauchy problem: Homogenization with corrector, Algebra i Analiz 21 (2009), no. 1, 3-60; English transl., St. Petersburg Math. J. 21 (2010), no. 1, 1-41. MR2553050(2010k:35035)

[K] T. Kato, Perturbation theory for linear operators, Grundlehren Math. Wiss., Bd. 132, SpringerVerlag New York, Inc., New York, 1966. MR0203473 (34:3324)

[Su1] T. A. Suslina, On the homogenization of periodic parabolic systems, Funktsional. Anal. i Prilozhen. 38 (2004), no. 4, 86-90; English transl., Funct. Anal. Appl. 38 (2004), no. 4, 309-312. MR2117512 (2005j:35008) 
[Su2] T. A. Suslina, Homogenization of periodic parabolic Cauchy problem, Nonlinear Equations and Spectral Theory, Amer. Math. Soc. Transl. Ser. 2, vol. 220, Amer. Math. Soc., Providence, RI, 2007, pp. 201-233. MR.2343612 (2008k:35030)

[Su3] Homogenization of a periodic parabolic Cauchy problem in the Sobolev space $H^{1}\left(\mathbb{R}^{d}\right)$, Math. Model. Nat. Phenom. 5 (2010), no. 4, 390-447. MR2662463 (2011g:35031)

[Su4] Homogenization of a parabolic Cauchy problem in the Sobolev class $H^{1}\left(\mathbb{R}^{d}\right)$, Funktsional. Anal. i Prilozhen. 44 (2010), no. 4, 91-96; English transl., Funct. Anal. Appl. 44 (2010), no. 4, 318-322. MR2768568

St. Petersburg State University, Department of Physics, Ul’yanovskaya 3, Petrodvorets, St. Petersburg 198504, Russia

E-mail address: vasilevskaya-e@yandex.ru

St. Petersburg State University, Department of Physics, Ul'yanovskaya 3, Petrodvorets, St. Petersburg 198504, Russia

E-mail address: suslina@list.ru

Received 30/JUN/2010

Translated by T. A. SUSLINA 